KIAS-P99085

hep-ph/9909429

\title{
One-loop Corrected Neutrino Masses and Mixing in the Supersymmetric Standard Model without R-parity
}

\author{
Eung Jin Chun and Sin Kyu Kang \\ Korea Institute for Advanced Study \\ 207-43 Cheongryangri-dong, Dongdaemun-gu, Seoul 130-012, Korea \\ Email addresses: ejchun@kias.re.kr, skkang@kias.re.kr
}

\begin{abstract}
We provide, in the R-parity violating supersymmetric standard model, a comprehensive analysis for the determination of sneutrino vacuum expectation values from the one-loop effective scalar potential, and also for one-loop renormalized neutrino masses and mixing by calculating the effective neutrino mass matrix in the weak basis. Applying our results to theories with gauge mediated supersymmetry breaking, we show how atmospheric and solar neutrino oscillations can be accommodated simultaneously in this framework. It is observed that the one-loop correction to sneutrino vacuum expectation values leads to a significant effect on the determination of the neutrino masses and mixing.
\end{abstract}

PACS number(s): 12.60.Jv, 11.30.Fs, 14.60.Pq 


\section{INTRODUCTION}

The minimal supersymmetric standard model (MSSM) may allow for explicit lepton number and thus R-parity violation through which neutrinos get nonzero masses and mixing [1]. As it is an attractive possibility to explain neutrino experiment data, there have been many works investigating neutrino masses from $\mathrm{R}$-parity violation [2]. In recent years, further development has been made to analyze three neutrino masses and mixing [3] accommodating the recent observation of the atmospheric muon neutrino oscillation in Super-Kamiokande [4] and other experimental data [5,6]. A very interesting feature of R-parity violation as the origin of neutrino masses and mixing is that this idea could be tested in future collider experiments despite the small R-parity violating couplings. Specifically, the mixing angles measured in the Super-Kamiokande [4] and the CHOOZ experiments [7] can be probed by detecting lepton number violating decay modes of the lightest supersymmetric particle [8,9].

In this paper, we basically extend the former studies in two ways. First, we calculate one-loop improved tree-level neutrino masses. In order to do that, we determine sneutrino vacuum expectation values $(\mathrm{VEVs})$ from the minimization of the one-loop effective scalar potential, which has usually been ignored in previous works \&. As will be shown later, the one-loop correction to sneutrino VEVs should not be ignored as it can drastically change the tree-level results. When there exists a cancellation among tree-level quantities in the determination of sneutrino VEVs, the loop contributions to the neutrino masses can be even larger than the tree ones. Furthermore, this phenomenon occurs generically in a certain parameter region. Secondly, we calculate one-loop renormalized neutrino masses and mixing utilizing the "effective mixing matrix" method [10]. As far as one concerns only the neutrino sector, this approach turns out to be very useful even though the results should be the same as in the usual on-shell renormalization scheme working in the tree-level mass basis [11,12]. Our procedure begins with constructing the $7 \mathrm{x} 7$ one-loop neutrino/neutralino mass matrix in the weak basis. Then, we obtain the seesaw-reduced 3x3 neutrino mass matrix from which physical neutrino masses and mixing are calculated. In this way, one can easily figure out the effects of each self-energy diagrams and simplify the calculation to obtain the main contributions. Our results are applied to the theories with gauge mediated supersymmetry breaking to discuss how large the one-loop results deviate from the tree-level ones. Finally, we show that the atmospheric and solar neutrino oscillations can be accommodated naturally in this framework. In particular, we present some typical examples for input parameters providing the vacuum or matter oscillation solution to the solar neutrino problem.

This paper is organized as follows. In Section II, we determine sneutrino VEVs by computing one-loop effective scalar potential, and present analytic expressions for all the one-loop contributions to sneutrino VEVs in Appendix A. In doing so, we also derive the diagonalization matrices for fermions or sfermions which get mixed further due to R-parity violation. In Section III, these diagonalization matrices will be used to calculate one-loop self-energy diagrams in the weak basis. This enables us to determine the dominant contributions to physical neutrino mass eigenvalues and mixing angles which could be relevant

\footnotetext{
${ }^{1}$ see however, Ref. [12] where diagrammatic method is used.
} 
for explaining the current neutrino data. The leading self-energy diagrams are calculated in Appendix B. In Section IV, we apply the above results to the R-parity violating theories with gauge mediated supersymmetry breaking and discuss the effects of one-loop corrections to sneutrino VEVs on the determination of the neutrino masses and mixing. Then, we illustrate how the atmospheric and solar neutrino data can be explained simultaneously in this framework. We conclude in Section V.

\section{SNEUTRINO VEVS FROM ONE-LOOP EFFECTIVE SCALAR POTENTIAL}

We start by writing the MSSM superpotential $W_{0}$ and $W_{1}$ conserving and violating R-parity/lepton number, respectively:

$$
\begin{aligned}
& W_{0}=\mu H_{1} H_{2}+h_{i}^{e} L_{i} H_{1} E_{i}^{c}+h_{i}^{d} Q_{i} H_{1} D_{i}^{c}+h_{i}^{u} Q_{i} H_{2} U_{i}^{c} \\
& W_{1}=\mu_{i} L_{i} H_{2}+\lambda_{i j k} L_{i} L_{j} E_{k}^{c}+\lambda_{i j k}^{\prime} L_{i} Q_{j} D_{k}^{c} .
\end{aligned}
$$

An unpleasant feature of R-parity violation is that there are too many free parameters essentially uncontrollable unless a certain theoretical framework for Yukawa hierarchies is invoked. For later use, we assume that $\lambda_{i} \equiv \lambda_{i 33}$ and $\lambda_{i}^{\prime} \equiv \lambda_{i 33}^{\prime}$ dominate over the other couplings for the first two generations, guided by the quark and lepton Yukawa hierarchy. The bilinear model where no trilinear R-parity violating couplings are present at the fundamental scale satisfies this assumption, as rotating away the bilinear terms generates the hierarchical trilinear couplings $\lambda, \lambda^{\prime}$.

One-loop effective scalar potential to determine the VEVs of the Higgs and sneutrino fields, $\left\langle H_{1,2}^{0}\right\rangle$ and $\left\langle L_{i}^{0}\right\rangle$ is given by

$$
V=\left[m_{L_{i} H_{1}}^{2} L_{i} H_{1}^{\dagger}+B_{i} L_{i} H_{2}+\text { h.c. }\right]+m_{L_{i}}^{2}\left|L_{i}\right|^{2}+V_{D}+V_{1}+\cdots
$$

where $V_{D}$ is the $S U(2) \times U(1)$ D-term, and $V_{1}=\frac{1}{64 \pi^{2}} \operatorname{Str} \mathcal{M}^{4}\left(\ln \frac{\mathcal{M}^{2}}{Q^{2}}-\frac{3}{2}\right)$ is the one-loop correction. In our notation, $m_{L_{i} H_{1}}^{2}$ contains not only the soft mass term but also the supersymmetric mass term $\mu \mu_{i}$ from the superpotential (1). Here we assume the R-parity violating parameters are small enough, $\mu_{i} \ll \mu$ and $m_{L_{i} H_{1}}^{2} \ll m_{L_{i}}^{2}$, etc., to produce light neutrinos of $m_{\nu}<1 \mathrm{eV}$, which would be a consequence of a horizontal symmetry [13. Furthermore, we take the universality among soft supersymmetry breaking terms at the scale of supersymmetry breaking where the bilinear term $\mu_{i} L_{i} H_{2}$ can be rotated away together with the corresponding soft terms. One of a natural framework for the universality is gauge mediated supersymmetry breaking which will be discussed in Section IV. Under these assumptions the intergenerational mixing terms like $\mu_{i} \mu_{i} L_{i} L_{j}^{\dagger}$ can be neglected. The minimization condition for the sneutrino fields, $\partial V / \partial L_{i}^{0}=0$, leads to

$$
\left\langle L_{i}^{0}\right\rangle=-\frac{B_{i}\left\langle H_{2}^{0}\right\rangle+m_{L_{i} H_{1}}^{2}\left\langle H_{1}^{0}\right\rangle+\Sigma_{L_{i}}^{(1)}\left\langle H_{1}^{0}\right\rangle}{m_{L_{i}}^{2}+\frac{1}{2} M_{Z}^{2} c_{2 \beta}+\Sigma_{L_{i}}^{(2)}},
$$

where $\Sigma_{L_{i}}^{(1,2)}$ are given by 


$$
\Sigma_{L_{i}}^{(1)}=\left.\frac{\partial V_{1}}{H_{1}^{0 *} \partial L_{i}^{0}}\right|_{0}, \quad \Sigma_{L_{i}}^{(2)}=\left.\frac{\partial V_{1}}{L_{1}^{0 *} \partial L_{i}^{0}}\right|_{0},
$$

where $\left.\right|_{0}$ means to put $L_{i}^{0}$ to zero. This expression is valid when R-parity violating parameters are small enough, $\mu_{i} \ll \mu$, etc.. In the case of small R-parity violation, the Higgs VEVs can be obtained from the usual minimization condition neglecting $\mathrm{R}$-parity violating couplings. The complete one-loop calculation for the Higgs VEVs can be found in Ref. 14.

R-parity violation mixes neutrinos, charged leptons, sleptons and sneutrinos with neutralinos, charginos, charged and neutral Higgses, respectively, and gives rise to more complicated and bigger mass matrices of fermions and sfermions. In the case of small R-parity violation, it is convenient to use first approximate seesaw diagonalization to rotate away the R-parity odd elements. Then one uses the further diagonalization process to obtain mass eigenvalues which contain the contributions from R-parity violating parameters up to their second order. It is now a simple manner to take derivatives of mass eigenvalues with respect to $L_{i}^{0}$ and find out the quantities in Eq. (四).

Let us follow the above prescription first to get charged slepton/Higgs contribution to sneutrino VEVs. Since sleptons and charged Higgs bosons get mixed, one has to diagonalize the $8 \times 8$ mass matrix for three slepton pairs and one Higgs pair which takes the form:

$$
\mathcal{M}^{2}=\left(\begin{array}{ll}
\mathcal{M}_{\tilde{l} l}^{2} & \mathcal{M}_{\tilde{l} H}^{2} \\
\mathcal{M}_{\tilde{l} H}^{2 \dagger} & \mathcal{M}_{H H}^{2}
\end{array}\right) .
$$

To calculate $\Sigma^{(1,2)}$ we keep the R-parity violating contributions to the above mass matrix up to quadratic terms in R-parity violating parameters. In this paper, we assume the absence of intergenerational mixing in slepton soft masses. However, there could arise in general Rparity violating intergenerational mixing masses which can be neglected as they give quartic contribution to mass eigenvalues. We further assume $\mathrm{CP}$ conservation and thus take the VEVs, $u_{i}=\left\langle L_{i}^{0}\right\rangle, v_{1,2}=\left\langle H_{1,2}^{0}\right\rangle$ to be real. Then, the submatrices of Eq. (5) in the weak basis $\left(\tilde{e}_{i L}, \tilde{e}_{i R}, H_{1}^{-}, H_{2}^{-}\right)$are given by

$$
\begin{aligned}
\mathcal{M}_{\tilde{l} l}^{2} & =\left(\begin{array}{cc}
M_{L i}^{2}-\left(g_{w}^{2}+\frac{1}{2} g_{z}^{2}\right) u^{2}+g_{w}^{2} u_{i}^{2}-2 h_{i}^{e} v_{1}\left(\lambda_{j} u_{j}\right) & M_{D i}^{2}+\delta_{i 3}\left(A_{j} u_{j}\right) \\
M_{D i}^{2}+\delta_{i 3}\left(A_{j} u_{j}\right) & M_{R i}^{2}+\left(g_{w}^{2}-g_{z}^{2}\right) u^{2}+\left(h_{i}^{e} u_{i}\right)^{2}-2 h_{i}^{e} v_{1}\left(\lambda_{j} u_{j}\right)
\end{array}\right) \\
\mathcal{M}_{\tilde{l} H}^{2} & =\left(\begin{array}{cc}
m_{L_{i} H_{1}}^{2}+\left(g_{w}^{2}-h_{i}^{e 2}\right) v_{1} u_{i} & -B_{i}+g_{w}^{2} u_{i} v_{2} \\
h_{i}^{e} v_{2} \mu_{i}+A_{i}^{e} u_{i} & h_{i}^{e} v_{1} \mu_{i}-h_{i}^{e} \mu u_{i}
\end{array}\right) \\
\mathcal{M}_{H H}^{2} & =\left(\begin{array}{cc}
M_{H_{1}^{-}}^{2}-\frac{1}{2}\left(2 g_{w}^{2}-g_{z}^{2}\right) u^{2}+h_{\tau}^{2} u_{3}^{2} & -B+g_{w}^{2} v_{1} v_{2} \\
-B+g_{w}^{2} v_{1} v_{2} & M_{H_{2}^{+}}^{2}+\frac{1}{2}\left(2 g_{w}^{2}-g_{z}^{2}\right) u^{2}
\end{array}\right),
\end{aligned}
$$

where $M_{L i}^{2}$, etc are the conventional R-parity conserving charged slepton or Higgs masses and summing over $j$ is understood for the quantities inside the parentheses in the first matrix, and we will take the lepton Yukawa couplings, $h_{1,2}^{e}=0$. Here we define the notation $g_{w}^{2} \equiv M_{W}^{2} / v^{2}$ and $g_{z}^{2} \equiv M_{Z}^{2} / v^{2}$ where $v^{2}=v_{1}^{2}+v_{2}^{2}+u^{2}$ and $u^{2}=\sum_{i} u_{i}^{2}$. Following the previous prescription, one can derive the charged slepton and Higgs contributions to $\Sigma^{(1,2)}$ as presented in Appendix A. Here we have to mention a few technical points. For diagonalizing away the R-parity odd matrix elements, we use the seesaw formula. That is, the matrix $\mathcal{M}$ of the form; 


$$
\mathcal{M}=\left(\begin{array}{cc}
M_{1} & \Delta \\
\Delta^{\dagger} & M_{2}
\end{array}\right)
$$

can be diagonalized by the "approximate" unitary matrix $\mathcal{U}$;

$$
\mathcal{U}=\left(\begin{array}{cc}
1-\frac{1}{2} \Theta \Theta^{\dagger} & -\Theta \\
\Theta^{\dagger} & 1-\frac{1}{2} \Theta^{\dagger} \Theta
\end{array}\right)
$$

if $M_{1,2}$ and $\Delta$ are submatrices satisfying $\Delta \ll M_{1,2}$. Being valid up to quadratic order in $\Delta$, the seesaw rotation matrix $\Theta$ is determined by the relation, $\Delta=M_{1} \Theta-\Theta M_{2}$ and the block diagonal matrices become

$$
\begin{aligned}
& M_{1} \rightarrow M_{1}+\frac{1}{2}\left(\Theta^{\dagger}+\Delta \Theta^{\dagger}\right) \\
& M_{2} \rightarrow M_{2}-\frac{1}{2}\left(\Theta^{\dagger} \Delta+\Delta^{\dagger} \Theta\right) .
\end{aligned}
$$

The essential step in the diagonalization is to decouple the Goldstone mode, $G^{-}$. This can be done by first going to the basis of vanishing sneutrino VEVs with the rotation matrix $\Theta_{G}$ and then applying the usual rotation $U_{\beta}$ with the angle $\beta$. Omitting the quadratic terms in R-parity violating parameters for simplicity, the combined rotation $\Theta_{G} U_{\beta}$ is given by

$$
\Theta_{G} U_{\beta}=\left(\begin{array}{cccc}
1 & 0 & a_{i} & 0 \\
0 & 1 & 0 & 0 \\
-a_{i} & 0 & 1 & 0 \\
0 & 0 & 0 & 1
\end{array}\right)\left(\begin{array}{cccc}
1 & 0 & 0 & 0 \\
0 & 1 & 0 & 0 \\
0 & 0 & -c_{\beta} & s_{\beta} \\
0 & 0 & s_{\beta} & c_{\beta}
\end{array}\right),
$$

where $a_{i} \equiv u_{i} / v_{1}$ are the values determined from tree level scalar potential. Note that in the full expression, the angle $\beta$ is determined by $\tan \beta=v_{2} / v_{1}^{\prime}$ where $v_{1}^{\prime}=\sqrt{v_{1}^{2}+u^{2}}$. Applying the above rotation to the charged lepton/Higgs mass matrix (5),60), one can explicitly see that there is a massless state up to the order of our expansion. Further diagonalization can be done by another seesaw rotation $\Theta_{\delta}$ which get rid of R-parity odd off-diagonal components, and the usual diagonalization $U_{e_{i}}$ of the $2 \times 2$ slepton mass matrix [see Appendix A]. Again in the leading approximation, $\Theta_{\delta} U_{e_{i}}$ is given by

$$
\Theta_{\delta} U_{e_{i}}=\left(\begin{array}{cccc}
1 & 0 & 0 & -\delta_{1}^{i} \\
0 & 1 & 0 & -\delta_{2}^{i} \\
0 & 0 & 1 & 0 \\
\delta_{1}^{i} & \delta_{2}^{i} & 0 & 1
\end{array}\right)\left(\begin{array}{cccc}
c_{e_{i}} & s_{e_{i}} & 0 & 0 \\
-s_{e_{i}} & c_{e_{i}} & 0 & 0 \\
0 & 0 & 1 & 0 \\
0 & 0 & 0 & 1
\end{array}\right)
$$

We obtain the following expressions for $\delta_{1,2}^{i}$;

$$
\begin{gathered}
M_{E_{i} H}^{2}=-a_{i} s_{\beta}\left(m_{H^{-}}^{2}-M_{W}^{2}\right)-\frac{B_{i}}{c_{\beta}}, \quad M_{E_{i}^{c} H}^{2}=\left(a_{i}-\frac{\mu_{i}}{\mu}\right) \frac{m_{i}^{e} \mu}{c_{\beta}} \\
\delta_{1}^{i}=\frac{M_{E_{i} H}^{2}\left(M_{R i}^{2}-m_{H^{-}}^{2}\right)-M_{E_{i}^{c} H^{2}}^{2} M_{D i}^{2}}{\left(m_{\tilde{e}_{i 1}}^{2}-m_{H^{-}}^{2}\right)\left(m_{\tilde{e}_{i 2}}^{2}-m_{H^{-}}^{2}\right)}, \quad \delta_{2}^{i}=\frac{M_{E_{i}^{c} H}^{2}\left(M_{L i}^{2}-m_{H^{-}}^{2}\right)-M_{E_{i} H^{2}}^{2} M_{D i}^{2}}{\left(m_{\tilde{e}_{i 1}}^{2}-m_{H^{-}}^{2}\right)\left(m_{\tilde{e}_{i 2}}^{2}-m_{H^{-}}^{2}\right)}
\end{gathered}
$$

In sum, the rotation matrix which brings the weak eigenstates $\left(\tilde{e}_{i L}, \tilde{e}_{i R}, H_{1}^{-}, H_{2}^{-}\right)$to the mass eigenstates $\left(\tilde{e}_{i 1}, \tilde{e}_{i 2}, H^{-}\right)$decoupling the Goldstone mode $G^{-}$is 


$$
\mathcal{U}^{C}=\left(\begin{array}{ccc}
c_{e_{i}} & s_{e_{i}} & -\delta_{1}^{i}+a_{i} s_{\beta} \\
-s_{e_{i}} & c_{e_{i}} & -\delta_{2}^{i} \\
\left(-a_{i}+\delta_{1}^{i} s_{\beta}\right) c_{e_{i}}-\delta_{2}^{i} s_{\beta} s_{e_{i}} & \left(-a_{i}+\delta_{1}^{i} s_{\beta}\right) s_{e_{i}}+\delta_{2}^{i} s_{\beta} c_{e_{i}} & s_{\beta} \\
\delta_{1}^{i} c_{\beta} c_{e_{i}}-\delta_{2}^{i} c_{\beta} s_{e_{i}} & \delta_{1}^{i} c_{\beta} s_{e_{i}}+\delta_{2}^{i} c_{\beta} c_{e_{i}} & c_{\beta}
\end{array}\right)
$$

This matrix will be used to compute one-loop self-energy diagrams for neutrino/neutralino masses.

Following the similar steps, we also obtain the CP-even and CP-odd sneutrino/Higgs contributions to sneutrino VEVs. The mass matrix in the weak basis $\left(\tilde{\nu}_{i}^{R}, H_{1}^{R}, H_{2}^{R}\right)$ of the real components of slepton and Higgs fields is

$$
\mathcal{M}_{\tilde{\nu}^{R} H}=\left(\begin{array}{ccc}
M_{\tilde{\nu}_{i}}^{2}+\frac{g_{z}^{2}}{2} u^{2}+g_{z}^{2} u_{i}^{2} & m_{L_{i} H_{1}}^{2}+g_{z}^{2} v_{1} u_{i} & B_{i}-g_{z}^{2} u_{i} v_{2} \\
m_{L_{i} H_{1}}^{2}+g_{z}^{2} v_{1} u_{i} & M_{H_{1}^{R}}^{2}+\frac{g_{z}^{2}}{2} u^{2} & B-g_{z}^{2} v_{1} v_{2} \\
B_{i}-g_{z}^{2} u_{i} v_{2} & B-g_{z}^{2} v_{1} v_{2} & M_{H_{2}^{R}}^{2}-\frac{g_{z}^{2}}{2} u^{2}
\end{array}\right)
$$

where $M_{\tilde{\nu}_{i}}^{2}$, etc are the usual R-parity conserving sneutrino/neutral Higgs boson masses. The diagonalization matrix in the leading expansion in terms of $\mathrm{R}$-parity violating parameters is

$$
\mathcal{U}^{S}=\left(\begin{array}{ccc}
1 & \zeta_{1}^{i} s_{\alpha}-\zeta_{2}^{i} c_{\alpha} & -\zeta_{1}^{i} c_{\alpha}-\zeta_{2}^{i} s_{\alpha} \\
\zeta_{1}^{i} & -s_{\alpha} & c_{\alpha} \\
\zeta_{2}^{i} & c_{\alpha} & s_{\alpha}
\end{array}\right)
$$

where $\alpha$ is the usual neutral Higgs scalar diagonalization angle [see Appendix A] and the seesaw rotation angle $\zeta_{1,2}^{i}$ is given by

$$
\begin{aligned}
\zeta_{1}^{i} & =-a_{i}+\frac{-a_{i}\left(m_{\tilde{\nu}_{i}}^{2}+M_{Z}^{2}\right) m_{A}^{2} s_{\beta}^{2}-B_{i} t_{\beta}\left(m_{\tilde{\nu}_{i}}^{2}+M_{Z}^{2} c_{2 \beta}\right)}{m_{\tilde{\nu}_{i}}^{4}-m_{\tilde{\nu}_{i}}^{2}\left(m_{h}^{2}+m_{H}^{2}\right)+m_{h}^{2} m_{H}^{2}} \\
\zeta_{2}^{i} & =\frac{a_{i}\left(m_{\tilde{\nu}_{i}}^{2}-M_{Z}^{2} c_{2 \beta}\right) m_{A}^{2} s_{2 \beta} / 2+B_{i}\left(m_{\tilde{\nu}_{i}}^{2}-M_{Z}^{2} c_{2 \beta}\right)}{m_{\tilde{\nu}_{i}}^{4}-m_{\tilde{\nu}_{i}}^{2}\left(m_{h}^{2}+m_{H}^{2}\right)+m_{h}^{2} m_{H}^{2}}
\end{aligned}
$$

As for the CP-odd sneutrino/Higgs fields, the mass matrix in the weak basis $\left(\tilde{\nu}^{I}, H_{1}^{I}, H_{2}^{I}\right)$ of the imaginary components of slepton and Higgs fields is

$$
\mathcal{M}_{\tilde{\nu}^{I} H}=\left(\begin{array}{ccc}
M_{\tilde{\nu}_{i}}^{2}+\frac{g_{z}^{2}}{2} u^{2} & m_{L_{i} H_{1}}^{2} & -B_{i} \\
m_{L_{i} H_{1}}^{2} & M_{H_{1}^{R}}^{2}+\frac{g_{z}^{2}}{2} u^{2} & -B \\
-B_{i} & -B & M_{H_{2}^{I}}^{2}-\frac{g_{z}^{2}}{2} u^{2}
\end{array}\right)
$$

where $M_{\tilde{\nu}_{i}^{I}}^{2}$, etc are the usual R-parity conserving sneutrino/neutral Higgs masses. As in the charged slepton/Higgs case, we first make the seesaw rotation, composed of $a_{i}=u_{i} / v_{1}$ to decouple the Goldstone mode, $G^{0}$. The diagonalization matrix which brings the weak eigenstates to the mass eigenstates $\left(\tilde{\nu}^{I}, A^{0}\right)$ becomes

$$
\mathcal{U}^{P}=\left(\begin{array}{cc}
1 & -\zeta_{3}^{i}+a_{i} s_{\beta} \\
-a_{i}+\zeta_{3}^{i} s_{\beta} & s_{\beta} \\
\zeta_{3}^{i} c_{\beta} & c_{\beta}
\end{array}\right),
$$


where the seesaw rotation angle $\zeta_{3}^{i}$ is given by

$$
\zeta_{3}^{i}=\frac{B_{i}-a_{i} B}{c_{\beta}\left(m_{A}^{2}-m_{\tilde{\nu}_{i}}^{2}\right)} .
$$

In Appendix A, we collect all of the information needed to calculate one-loop corrected sneutrino VEVs and present complete analytic results. For the numerical calculation, we take the renormalization scale, $Q^{2}=m_{\tilde{t}_{1}} m_{\tilde{t}_{2}}$, around which the one-loop contribution $V_{1}$ is minimized. The mixing matrices given in Eqs. (13), (15) and (18) play a role of inducing the effective $\lambda$ or $\lambda^{\prime}$ couplings in the mass basis and will be used to calculate the one-loop neutrino masses. We also need the neutrino/neutralino and charged lepton/chargino mixing matrices [15,9] which we summarize in Appendix A for completeness.

\section{ONE-LOOP CORRECTED NEUTRINO MASS MATRIX}

The neutrino/neutralino mass matrix at one-loop level is given by

$$
M^{\text {pole }}\left(p^{2}\right)=M(Q)+\Pi\left(p^{2}\right)-\frac{1}{2}\left(M(Q) \Sigma\left(p^{2}\right)+\Sigma\left(p^{2}\right) M(Q)\right)
$$

where $Q$ is the renormalization scale, and $M(Q)$ is the the $\overline{D R}$ renormalized $7 \times 7$ tree-level mass matrix, $\Pi$ and $\Sigma$ are the contributions from one-loop self-energy diagrams. To compute the one-loop corrected mass matrix (20), we work in the weak basis, that is, we evaluate $\Pi$ 's and $\Sigma$ 's with weak eigenstate neutrino/neutralino on external legs and mass eigenstates running inside loops. For this, we express the propagators in the loops in terms of the mass eigenstates as calculated in Appendix B. A complete one-loop analysis has been performed in Refs. [11,12] where the mass matrix (20) is calculated in the tree-level mass basis and mass eigenvalues and mixing matrix are obtained by re-diagonalizing the one-loop mass matrix. In our approach, it is useful to make a few observations which facilitate out calculation. Let us recast the mass matrix (20) as

$$
\begin{aligned}
M^{\text {pole }}\left(p^{2}\right)= & \left(\begin{array}{cc}
0 & M_{D} \\
M_{D}^{T} & M_{N}
\end{array}\right)(Q)+\left(\begin{array}{cc}
\Pi_{n} & \Pi_{D} \\
\Pi_{D}^{T} & \Pi_{N}
\end{array}\right)\left(p^{2}\right) \\
& -\frac{1}{2}\left(\begin{array}{cc}
M_{D} \Sigma_{D}^{T}+\Sigma_{D} M_{D}^{T} & \Sigma_{n} M_{D}+M_{D} \Sigma_{N}+\Sigma_{D} M_{N} \\
M_{D}^{T} \Sigma_{n}+\Sigma_{N} M_{D}^{T}+M_{N} \Sigma_{D}^{T} & M_{N} \Sigma_{N}+\Sigma_{N} M_{N}
\end{array}\right)\left(p^{2}\right)
\end{aligned}
$$

where neutrino-neutralino mixing elements of $M_{D}$ or $\Pi_{D}, \Sigma_{D}$ are much smaller than $M_{N}$ or $\Pi_{N}, \Sigma_{N}$ by factor of $\mathcal{O}\left(a_{i}\right)$. If we are interested only in neutrino mass eigenvalues and mixing matrix, it is convenient to obtain first the seesaw reduced $3 \times 3$ neutrino mass matrix. Applying seesaw formula to Eq. (21) and keeping one-loop order terms, we find the effective $3 \mathrm{x} 3$ neutrino mass matrix as follows;

$$
\begin{aligned}
M^{\nu}\left(p^{2}\right)= & -M_{D} M_{N}^{-1} M_{D}^{T}(Q)+M_{D} M_{N}^{-1} \Pi_{N}\left(p^{2}\right) M_{N}^{-1} M_{D}^{T} \\
& +\left(M_{D} M_{N}^{-1} M_{D}^{T} \Sigma_{n}\left(p^{2}\right)+\Sigma_{n}\left(p^{2}\right) M_{D} M_{N}^{-1} M_{D}^{T}\right) \\
& +\left(M_{D} M_{N}^{-1} \Pi_{D}^{T}\left(p^{2}\right)+\Pi_{D}\left(p^{2}\right) M_{N}^{-1} M_{D}^{T}\right)+\Pi_{n}\left(p^{2}\right)
\end{aligned}
$$


As neutrino masses are negligible, we can now take $p^{2}=0$ without ambiguity to calculate physical neutrino masses and mixing. Our procedure is in fact identical to that developed in Ref. [10] where the mixing renormalization is performed by utilizing the $Q$-independent "effective mixing matrix". The effective mixing matrix $U^{\text {eff }}$ is calculated from the diagonalization matrix $U\left(p^{2}\right)$ of the mass matrix $M^{\text {pole }}\left(p^{2}\right)$ in the weak basis (20) by taking $U_{i j}^{e f f}=U_{i j}\left(m_{j}^{2}\right)$. According to the results of [10], this method has several nice features. First, one can avoid superficial singularity reflecting the arbitrariness in the diagonalization of the mass matrix with degenerate massless neutrinos at tree level. Second, as far as neutrino mixing is concerned, the mixing matrix $U^{\nu}=U\left(p^{2} \rightarrow 0\right)$ determined from the diagonalization of the effective $3 \times 3$ matrix (22) can be considered as the full unitary mixing matrix, up to a negligible correction of $\mathcal{O}\left(a_{i}^{2}\right)$. Furthermore, the scale dependence which appears only in $\Sigma_{n}$ turns out to be very small as we will discuss below.

Note that the first term in Eq. (22) is the tree-level mass which makes only one neutrino massive. The other terms show one-loop contributions from various self-energy diagrams. It is interesting to see that $\Sigma_{D, N}$ do not appear in $M^{\nu}\left(p^{2}\right)$ and thus one does not have to calculate them. We can make further simplifications. First, in the basis where $\mu_{i}$ terms are rotated away, not only the tree-level mass matrix but also the loop mass matrix in Eq. (22) take a factorized form $M_{i j}^{\nu} \propto a_{i} a_{j}$ [9], That is, the contribution from $\Pi_{N}$ is aligned with the tree-level mass matrix and thus gives overall correction to the dominant tree-level mass in the direction $\sim a_{i} \nu_{i}$. One-loop correction to neutralino masses has been analyzed in Ref. [16], according to which the $\Pi_{N}$ contribution typically gives $6 \%$ correction to the tree-level values. As we are interested in the leading correction to the masses of the two light neutrinos, we will ignore this correction by working in the basis with $\mu_{i}=0$. Secondly, once no flavor changing sfermion masses are assumed, $\Sigma_{n}$ has only diagonal elements which produces also aligned correction of the order $\alpha_{2} / 4 \pi \lesssim 1 \%$, and thus we can also ignore this contribution. Then we are left with the last two one-loop corrections involving $\Pi_{n}$ and $\Pi_{D}$ in Eq. (22) which will give rise to a sizable contribution to neutrino masses and mixing. Therefore, the resulting neutrino mass matrix is

$$
\begin{aligned}
M_{i j}^{\nu} & =\frac{M_{Z}^{2}}{F_{N}} \xi_{i} \xi_{j} c_{\beta}^{2}+\frac{M_{Z}^{2}}{F_{N}}\left(\xi_{i} \eta_{j}+\eta_{i} \xi_{j}\right) c_{\beta}+\Pi_{\nu_{i} \nu_{j}} \quad \text { where } \\
F_{N} & =-\left(M_{1} M_{2} / M_{\tilde{\gamma}}+M_{Z}^{2} s_{2 \beta} / \mu\right), \quad M_{\tilde{\gamma}}=M_{1} c_{W}^{2}+M_{2} s_{W}^{2} \\
\eta_{i} & =\Pi_{\nu_{i} \tilde{B}^{0}}\left(\frac{-M_{2} s_{W}^{2}}{M_{\tilde{\gamma}} M_{W} t_{W}}\right)+\Pi_{\nu_{i} \tilde{W}_{3}}\left(\frac{M_{1} c_{W}^{2}}{M_{\tilde{\gamma}} M_{W}}\right)+\Pi_{\nu_{i} \tilde{H}_{1}^{0}}\left(\frac{s_{\beta}}{\mu}\right)+\Pi_{\nu_{i} \tilde{H}_{2}^{0}}\left(\frac{-c_{\beta}}{\mu}\right)
\end{aligned}
$$

Note that $\xi_{i} \equiv a_{i}-\mu_{i} / \mu$ becomes $a_{i}$ in the basis with $\mu_{i}=0$. Let us now make a qualitative discussion on various contributions to $\eta_{i}$ and $\Pi_{\nu_{i} \nu_{j}}$.

The one-loop diagrams contributing to $\Pi_{D}\left(\right.$ or $\left.\Pi_{\nu \psi^{0}}\right)$ can be divided into [DD], [DF] and $[\mathrm{FF}]$ types which involve two gauge vertices, one gauge and one Yukawa vertices, and two Yukawa vertices 国. The explicit formulae for $\Pi_{D}$ (and also $\Pi_{n}$ ) are presented in Appendix B. The [DD] type contains diagrams with $Z / W$, sneutrino/Higgs, charged slepton/ Higgs exchanges. Among them, the Z/W exchanging diagrams give corrections of $\mathcal{O}\left(\frac{\alpha_{2}}{4 \pi} \frac{m_{\tau}^{2} t_{\beta}}{M_{W}^{2}}\right) \lesssim 10^{-4}$ which are aligned with the tree level masses and thus can be safely ignored. The contribution from the charged slepton/Higgs exchange diagrams (involving also charged lepton/chargino 
exchange) can be as large as $\frac{\alpha_{2}}{4 \pi} t_{\beta}$ apart from the possible alignment as can be read from mixing matrices $(12,13)$ and (A22, A23). The same is true for the sneutrino/Higgs exchanging diagrams (involving also neutrino/neutralino exchange). From our explicit calculation in the next section, we will see that the seemingly large correction of order $\frac{\alpha_{2}}{4 \pi} t_{\beta}$ for large $\tan \beta$ is compensated by a large suppression factor and thus $\xi_{i} \gg \eta_{i}$. The [DF] type includes the diagrams with $h_{\tau}$ coupling involving charged slepton/Higgs exchange, $\lambda$ coupling involving stau/tau exchange, and $\lambda^{\prime}$ coupling involving sbottom/bottom exchange. The [DF] diagrams with $h_{\tau}$ coupling can be negligible for small $\tan \beta$ as the correction is of the order $\frac{g h_{\tau}}{16 \pi^{2}} \frac{m_{\tau}}{M_{W}} \lesssim 10^{-5}$ and are sub-leading to the corresponding diagrams contributing to $\Pi_{n}$ for large $\tan \beta$. In the $[\mathrm{FF}]$ type, there are diagrams with $h_{\tau} h_{\tau}, h_{\tau} \lambda$ and $h_{b} \lambda^{\prime}$ couplings. Generically, these diagrams are sub-leading to the similar ones in $\Pi_{n}$.

There are also $[\mathrm{DD}],[\mathrm{DF}]$, and $[\mathrm{FF}]$ types in the $\Pi_{n}\left(\right.$ or $\left.\Pi_{\nu \nu}\right)$ diagrams. The [DD] type can be ignored as the $\mathrm{Z}$ exchanging diagrams give aligned corrections of the order of $1 \%$ and the sneutrino exchanging ones are also negligible. In the [DF] type, there are diagrams with the coupling $h_{\tau}$ and $\lambda$. The first ones give rise to the correction $\sim \frac{g h_{\tau}}{16 \pi^{2}} \frac{m_{\tau} t_{\beta}}{M_{W}}$ which has $\tan \beta$ enhancement compared to the corresponding diagrams in $\Pi_{D}$ as mentioned before. In the $[\mathrm{FF}]$ type, there are diagrams involving $h_{\tau} h_{\tau}, h_{\tau} \lambda, \lambda \lambda$ and $\lambda^{\prime} \lambda^{\prime}$ couplings. The first two classes of diagrams may have significant contributions for large $\tan \beta$ as noted in Refs. [21, which will be partly confirmed in the next section. The last two classes of diagrams are the frequently considered ones as they typically give the main contributions to the one-loop correction.

Our qualitative discussion on the one-loop corrected neutrino masses will be confirmed by numerical computation performed in the next section. For our explicit computation, we use gauge-mediated supersymmetry breaking models [17] where the universality arises more naturally than in supergravity models. A rigorous calculation in minimal supergravity models with bilinear terms has been performed in Ref. [12].

\section{ATMOSPHERIC AND SOLAR NEUTRINO OSCILLATIONS IN GAUGE-MEDIATED SUPERSYMMETRY BREAKING MODELS}

In this section, we discuss the properties of the one-loop improved neutrino mass matrix obtained in the previous section through explicit calculation. Then, our emphasis is put on addressing whether or how the atmospheric and solar neutrino masses and mixing angles can be realized in the context of R-parity violating models with supersymmetry broken by the gauge mediation mechanism. In a gauge mediation model, soft parameters of sfermion fields depend only on their gauge quantum numbers upon supersymmetry breaking, and thus the supersymmetric flavor problem is resolved in a natural way [18. However, a difficulty in this type of models is that the origin of $\mu$ and $B$ terms cannot be provided in its own context. This also applies to the R-parity violating bilinear terms $\mu_{i}, B_{i}$. Assuming, however, $B$ and $B_{i}$ terms come from the same origin, we take $B / B_{i}=\mu / \mu_{i}$. Then, one can rotate away the R-parity violating bilinear terms into the Higgs bilinear term at the mediation scale and thus no sneutrino VEVs can be generated. But this property is spoiled by renormalization group evolution and thus sneutrinos get nonzero VEVs at the weak scale. We solve renormalization group equation to determine these sneutrino VEVs for given input parameters $\mu_{i}$ 
or $\lambda^{\prime}, \lambda$. In the R-parity preserving case, an extensive study of sparticle spectrum through renormalization group evolution has been done in Ref. [19]. In this work, we take a minimal gauge mediation model assuming one pair of messenger multiplets $(\mathbf{5}+\overline{\mathbf{5}})$ [17 and put the messenger scale equal to the messenger sector supersymmetry breaking scale $\Lambda$. In most cases, we will set $\Lambda=75 \mathrm{TeV}$.

The simplest R-parity violating model is the "bilinear model" in which only bilinear R-parity violating terms are present at the fundamental scale, or at the mediation scale of supersymmetry breaking in our case. This model having only three R-parity violating parameters is more predictive and turns out to be very restrictive as we will show. It is also conceivable that there are both bilinear and trilinear couplings originated from a certain flavor symmetry which also explains quark and lepton mass hierarchies [13]. In this spirit, we assume that $\lambda_{i}=\lambda_{i 33}$ and $\lambda_{i}^{\prime}=\lambda_{i 33}^{\prime}$ are much larger than the other couplings and give rise to experimentally relevant contributions. We call this the "trilinear model" which has five R-parity violating parameters (note that $\lambda_{3} \equiv 0$ ), as the bilinear terms $\mu_{i}$ can be rotated away into the trilinear couplings. For our purpose, it is then convenient to use renormalization group equations in the basis where the bilinear terms $\mu_{i}$ are rotated away at all the scales [20], which are recollected in Appendix C.

Before investigating realistic solutions to neutrino problems, it is worth discussing how much one-loop corrections can change tree-level results. As an example, we take a bilinear model with $\mu_{1}=\mu_{2}=\mu_{3}=10^{-4} \mu$ at the mediation scale $\Lambda=75 \mathrm{TeV}$. By rotating away $\mu_{i}$, we have

$$
\lambda_{i}^{\prime}=\frac{\mu_{i}}{\mu} h_{b}, \quad \lambda_{i}=\frac{\mu_{i}}{\mu} h_{\tau} .
$$

In Table I, we show the values of R-parity violating parameters and one-loop contributions to sneutrino VEVs calculated at $Q^{2}=m_{\tilde{t}_{1}} m_{\tilde{t}_{2}}$. In the table, $\tilde{q}, \phi^{-}, S^{0}, P^{0}, \psi^{0}, \psi^{-}$, and $Z / W$ denote the squark, charged slepton/Higgs, CP-even and CP-odd sneutrino/Higgs, neutrino/neutralino, charged lepton/chargino and gauge boson contributions to $\Sigma_{L_{i}}$, respectively. The main contribution to $\Sigma^{(1)}$ comes from stops or sbottoms, and we do not show vanishing or negligible contributions from $\psi^{0}$ or bottom quark, etc. For small $\tan \beta, \Sigma^{(1)}$ gives about $18 \%$ correction to the tree-level quantity, $B_{i} t_{\beta}+m_{L_{i} H_{1}}$ [see Eq. (33)]. The dominant contribution to $\Sigma^{(2)}$ comes from charged slepton/Higgs. Contrary to the case of $\Sigma^{(1)}$, the squark contribution is negligible as there is an almost precise cancellation between uptype and down-type squark contributions. The correction $\Sigma^{(2)}$ to $m_{\tilde{\nu}}^{2}$ is typically small and $5 \%$ in our case. Taking both one-loop contributions $\Sigma^{(1,2)}$ into account, the one-loop improved sneutrino VEVs, $\xi_{i}$, get increased by $13 \%$ from the tree-level value $\xi_{i}^{0}$ for $\tan \beta=3$. This corresponds to a $27 \%$ increase of the tree-level neutrino mass as it is proportional to $\xi_{i}^{2}$. This is a significant change when one tries to explain the experimental data. More seriously, the one-loop correction could even upset the tree-level calculation. As can be seen from Table I with $\tan \beta=30$, the one-loop improved value $\xi_{i}$ is about 7 times larger than the tree-level value $\xi_{i}^{0}$. This phenomenon occurs due to a cancellation between the tree-level quantities. As one can see from Table I, even though each quantity $m_{L_{i} H_{1}}^{2}$ or $B_{i} t_{\beta}$ is about 10 times larger than the one-loop correction $\Sigma^{(1)}$, there exists a cancellation of first two digits in the sum, $B_{i} t_{\beta}+m_{L_{i} H_{1}}$. Therefore, it is essential to include one-loop corrections in the calculation of neutrino masses and mixing arising from R-parity violation. 
To show the $\tan \beta$ dependence of tree and loop masses, we present order of magnitudes of some mass matrix components in the last five rows of Table I. Let us first note that while $\eta_{i}$ get increased mildly with $\tan \beta$, the components of $\Pi_{n}$ with $h_{\tau} \lambda_{i}$ couplings get increased tremendously with $\tan \beta$ and become comparable to the contributions from $\lambda \lambda$ couplings, as was first noted in Ref. [21]. While the heaviest neutrino mass dominated by the tree-level value gets little changed, the second largest neutrino mass strongly depends on $\tan \beta$.

Let us now turn to an important question whether a realistic neutrino mass matrix can be obtained in our scheme. We first discuss the bilinear model. As can be seen in Table I, a bilinear model with low $\tan \beta$ produces two heavy neutrino masses in the right range for the atmospheric and solar neutrino vacuum oscillation mass scale. But, it appears to be impossible to obtain bi-large mixing required in this case. In order to have bi-large mixing, one needs $U_{13}^{\nu} \ll 1$ which means $\xi_{1} \ll \xi_{2,3}$ as the tree-level mass dominates [9]. This implies, in turn, $\mu_{1} \ll \mu_{2,3}$ which gives rise to the solar neutrino mixing angle $\sim \mu_{1} / \mu_{2,3}$, leading to a contradiction. On the other hand, one can easily find a small mixing MSW solution to solar neutrino problem 22 for a reasonably large $\tan \beta$. A solution is as follows. Let us take the input values with $\tan \beta=24$;

$$
\frac{\mu_{1}}{\mu}=6 \times 10^{-6}, \quad \frac{\mu_{2}}{\mu}=\frac{\mu_{3}}{\mu}=1.5 \times 10^{-4} .
$$

This gives rise to the neutrino mass matrix in the eV unit;

$$
\begin{aligned}
& -\left(\begin{array}{ccc}
5.7 \times 10^{-5} & 1.4 \times 10^{-3} & 1.3 \times 10^{-3} \\
1.4 \times 10^{-3} & 3.6 \times 10^{-2} & 3.2 \times 10^{-2} \\
1.3 \times 10^{-3} & 3.2 \times 10^{-2} & 2.9 \times 10^{-2}
\end{array}\right)+\left(\begin{array}{lll}
2.2 \times 10^{-5} & 5.5 \times 10^{-4} & 2.3 \times 10^{-4} \\
5.5 \times 10^{-4} & 1.4 \times 10^{-2} & 5.8 \times 10^{-3} \\
2.3 \times 10^{-4} & 5.8 \times 10^{-3} & 2.5 \times 10^{-3}
\end{array}\right) \\
& =-\left(\begin{array}{lll}
3.5 \times 10^{-5} & 8.7 \times 10^{-4} & 1.1 \times 10^{-3} \\
8.7 \times 10^{-4} & 2.2 \times 10^{-2} & 2.6 \times 10^{-2} \\
1.1 \times 10^{-3} & 2.6 \times 10^{-2} & 2.6 \times 10^{-2}
\end{array}\right)
\end{aligned}
$$

where the first matrix on the left-hand side is the tree-level mass and the second one is the one-loop mass. This leads to the mass eigenvalues and oscillation amplitude;

$$
\begin{aligned}
& m_{1}=4.6 \times 10^{-19}, \quad m_{2}=2.3 \times 10^{-3}, \quad m_{3}=5.1 \times 10^{-2} \\
& \sin ^{2} 2 \theta_{\text {sol }}=0.0035, \quad \sin ^{2} 2 \theta_{\text {atm }}=0.99, \quad \sin ^{2} 2 \theta_{\text {chooz }}=0.0029 .
\end{aligned}
$$

In our notation, $\sin ^{2} 2 \theta_{\text {sol }}$ is the $\nu_{e}$ disappearance oscillation amplitude corresponding to $\Delta m_{21}^{2}$, and $\sin ^{2} 2 \theta_{\text {atm }}\left(\sin ^{2} 2 \theta_{\text {chooz }}\right)$ is the $\nu_{\mu} \rightarrow \nu_{\tau}$ ( $\nu_{e}$ disappearance) oscillation amplitudes corresponding to $\Delta m_{32,31}^{2}$, which are measured in the solar neutrino [5], and the atmospheric neutrino [4] (CHOOZ [7]) experiments, respectively. Let us note that such a solution can be obtained in a limited range of $\tan \beta=22-26$. If one reduces the mediation scale, a larger $\tan \beta$ is needed to achieve this kind of solution; e.g., $\tan \beta \approx 40$ for $\Lambda=50 \mathrm{TeV}$. Our investigation shows that the small mixing MSW solution to the solar neutrino problem can be obtained for rather smaller $\tan \beta$ than claimed in Ref. 21]. The main reason for this discrepancy is that the possibility of a precise cancellation for large $\tan \beta$ discussed above was not considered legitimately in a qualitative analysis of Ref. [21]. In fact, for $\tan \beta>30$ (with $\Lambda=75 \mathrm{TeV}$ ), the loop corrections get weird due to a large third generation effect 
$\left(h_{b}, h_{\tau} \sim 1\right)$ so that the input, $\mu_{2} \approx \mu_{3}$, motivated by large atmospheric neutrino mixing, does not work at all.

In trilinear models, we can have more freedom and thus find more variety of solutions. A remarkable result is that the bi-maximal mixing solution for the atmospheric and solar neutrino vacuum oscillations [23] can be obtained in our framework. For $\tan \beta=2$, a set of the input values to provide bi-maximal mixing is

$$
\begin{aligned}
& \lambda_{1}=2.3 \times 10^{-6}, \quad \lambda_{2}=7.7 \times 10^{-6}, \\
& \lambda_{1}^{\prime}=-9.4 \times 10^{-7}, \quad \lambda_{2}^{\prime}=\lambda_{3}^{\prime}=-1.3 \times 10^{-5} .
\end{aligned}
$$

A key feature to find such a solution is that one needs $\lambda_{1}^{\prime}$ smaller than $\lambda_{2,3}^{\prime}$ by about one order of magnitude to yield $U_{13}^{\nu} \ll 1$ and rather large $\lambda_{1}$ to increase the mass components in the $\nu_{e}$ direction. The resultant mass matrix is given by

$$
\begin{gathered}
-\left(\begin{array}{ccc}
1.3 \times 10^{-4} & 1.8 \times 10^{-3} & 1.7 \times 10^{-3} \\
1.8 \times 10^{-3} & 2.7 \times 10^{-2} & 2.5 \times 10^{-2} \\
1.7 \times 10^{-3} & 2.5 \times 10^{-2} & 2.3 \times 10^{-2}
\end{array}\right)+\left(\begin{array}{ccc}
6.7 \times 10^{-6} & 2.9 \times 10^{-5} & 1.6 \times 10^{-5} \\
2.9 \times 10^{-5} & 1.1 \times 10^{-4} & 5.0 \times 10^{-5} \\
1.6 \times 10^{-5} & 5.0 \times 10^{-5} & -2.2 \times 10^{-6}
\end{array}\right) \\
=-\left(\begin{array}{ccc}
1.2 \times 10^{-4} & 1.8 \times 10^{-3} & 1.7 \times 10^{-3} \\
1.8 \times 10^{-3} & 2.7 \times 10^{-2} & 2.5 \times 10^{-2} \\
1.7 \times 10^{-3} & 2.5 \times 10^{-2} & 2.3 \times 10^{-2}
\end{array}\right)
\end{gathered}
$$

leading to the following mass eigenvalues and oscillation amplitudes;

$$
\begin{aligned}
& m_{1}=2.4 \times 10^{-6}, \quad m_{2}=8.1 \times 10^{-6}, \quad m_{3}=5.0 \times 10^{-2} \\
& \sin ^{2} 2 \theta_{\text {sol }}=0.97, \quad \sin ^{2} 2 \theta_{\text {atm }}=0.99, \quad \sin ^{2} 2 \theta_{\text {chooz }}=0.0098 .
\end{aligned}
$$

In this case, we have $\Delta m_{21}^{2}=0.6 \times 10^{-10} \mathrm{eV}^{2}$. Compared to the other solutions, this is rather sensitive to the input values, but one does not need to rely on fine-tuning of the input parameters.

Our last example is to accommodate the small mixing MSW solar neutrino solution even for low $\tan \beta$. It is the case where $\lambda_{i}$ is larger than $\lambda_{i}^{\prime}$. Specifically, the input values with $\tan \beta=5$;

$$
\begin{array}{ll}
\lambda_{1}=1.6 \times 10^{-6}, & \lambda_{2}=4.0 \times 10^{-5} \\
\lambda_{1}^{\prime}=4.9 \times 10^{-7}, & \lambda_{2}^{\prime}=\lambda_{3}^{\prime}=1.2 \times 10^{-5},
\end{array}
$$

give rise to the mass matrix;

$$
\begin{aligned}
& -\left(\begin{array}{ccc}
4.0 \times 10^{-5} & 1.0 \times 10^{-3} & 8.5 \times 10^{-4} \\
1.0 \times 10^{-3} & 2.5 \times 10^{-2} & 2.1 \times 10^{-2} \\
8.5 \times 10^{-4} & 2.1 \times 10^{-2} & 1.8 \times 10^{-2}
\end{array}\right)+\left(\begin{array}{ccc}
4.4 \times 10^{-6} & 1.1 \times 10^{-4} & -7.5 \times 10^{-6} \\
1.1 \times 10^{-4} & 2.7 \times 10^{-3} & -1.9 \times 10^{-4} \\
-7.5 \times 10^{-6} & -1.9 \times 10^{-4} & -1.4 \times 10^{-6}
\end{array}\right) \\
& =-\left(\begin{array}{lll}
3.6 \times 10^{-5} & 8.9 \times 10^{-4} & 8.5 \times 10^{-4} \\
8.9 \times 10^{-4} & 2.2 \times 10^{-2} & 2.1 \times 10^{-2} \\
8.5 \times 10^{-4} & 2.1 \times 10^{-2} & 1.8 \times 10^{-2}
\end{array}\right) \text {. }
\end{aligned}
$$

The corresponding mass eigenvalues and oscillation amplitudes are 


$$
\begin{aligned}
& m_{1}=1.5 \times 10^{-20}, \quad m_{2}=1.4 \times 10^{-3}, \quad m_{3}=4.2 \times 10^{-2} \\
& \sin ^{2} 2 \theta_{\text {sol }}=0.0029, \quad \sin ^{2} 2 \theta_{\text {atm }}=0.99, \quad \sin ^{2} 2 \theta_{\text {chooz }}=0.0035 .
\end{aligned}
$$

In this solution, the loop mass matrix is dominated by the diagrams with $\lambda \lambda$ couplings and the other diagrams can be neglected. In all the realistic solutions, we find that the mass matrix elements coming from the diagrams with $h_{\tau} \lambda$ and $h_{b} \lambda^{\prime}$ in $\Pi_{D}$, and with $g h_{\tau}$ and $h_{\tau} h_{\tau}$ in $\Pi_{D, n}$ are smaller than the others by more than one order of magnitude. Furthermore, the $\Pi_{D}$ diagrams can be omitted as they give negligible contributions to the resulting mass eigenvalues and mixing angles. Among the $\Pi_{n}$ diagrams, one should not ignore the diagrams with $g \lambda, h_{\tau} \lambda$ in the parameter region with large $\tan \beta$, or relatively large $\lambda$ (as in the first two solutions), as their contributions become comparable to those with $\lambda \lambda$ or $\lambda^{\prime} \lambda^{\prime}$ which usually give largest contributions.

\section{CONCLUSIONS}

We have performed a comprehensive analysis on the one-loop corrected neutrino masses and mixing. Our first concern was to determine sneutrino vacuum expectation values at the one-loop level by employing one-loop effective scalar potential. R-parity violation mixes R-parity even and odd particles and thus sfermion and fermion mass matrices are more complicated than in the R-parity conservation case. However, under the condition that R-parity violating couplings are small as implied by small neutrino masses, one can make an analytic diagonalization up to the quadratic order in R-parity violating parameters to compute the complete one-loop contributions to sneutrino minimization condition. The results are presented in Appendix A. This one-loop determination of sneutrino VEVs is essential to confront the R-parity violating models with experimental data. It has been observed that the one-loop correction can give rise to a significant change from the tree-level calculation, in particular, when there occurs cancellation between tree-level contributions. Taking gauge-mediated supersymmetry breaking models, we have shown that this kind of cancellation arises naturally for large $\tan \beta$ and the one-loop correction can change even the order of magnitude of the heaviest neutrino mass.

We have then considered one-loop renormalization of neutrino mass matrix to determine mass eigenvalues and mixing angles for all three neutrinos. Concerning the neutrino sector only, it turns out that the use of the $Q$-independent effective mixing matrix is very useful to calculate one-loop corrected neutrino masses and mixing. In this procedure, we compute $7 \times 7$ one-loop neutrino/neutralino mass matrix in the weak basis, and make a $Q$-independent seesaw diagonalization to obtain $3 \times 3$ effective neutrino mass matrix. From this effective mass matrix, one can simply calculate mass eigenvalues and mixing matrix working at zero momentum $p^{2}=0$. It is then argued that this procedure simplifies the calculation in the basis of $\mu_{i}=0$ if one wants to calculate the leading contributions which are summarized in Appendix B.

Following the above procedures, we have investigated the properties of neutrino mass matrix to see whether the atmospheric and solar neutrino oscillations can be obtained in the framework of R-parity violating theories with gauge mediated supersymmetry breaking. In

the bilinear model, together with the atmospheric neutrino oscillation, one can only obtain 
the small mixing MSW solution to the solar neutrino problem taking large $\tan \beta \gtrsim 20$. For the case of the trilinear model, one can have more solutions. With low tan $\beta$, the bimaximal mixing solution to the atmospheric and solar neutrino problems can be realized. Furthermore, varying 5 free parameters, it has been shown that the small mixing MSW solution can be obtained even for small $\tan \beta$. We would like to emphasize that all of these R-parity violating solutions to neutrino problems can be realized without any fine-tuning, apart from the smallness of R-parity violating parameters, $\mu_{i}, \lambda$ and $\lambda^{\prime}$, which may be originated from a certain flavor symmetry explaining the quark and lepton mass hierarchies.

\section{APPENDIX A: ONE-LOOP CONTRIBUTIONS TO SNEUTRINO VEVS}

We collect here one-loop contributions to sneutrino minimization condition (3) by calculating all the field-dependent particle masses.

Stop contribution: The stop mass matrix including $\mathrm{R}$-parity violating contribution is given by

$$
\mathcal{M}_{\tilde{t}}^{2}=\left(\begin{array}{cc}
m_{Q_{3}}^{2}+m_{t}^{2}+\frac{1}{6}\left(4 M_{W}^{2}-M_{Z}^{2}\right) c_{2 \beta} & A_{t} v_{2}+h_{t}\left(\mu v_{1}+\mu_{i} u_{i}\right) \\
A_{t} v_{2}+h_{t}\left(\mu v_{1}+\mu_{i} u_{i}\right) & m_{\tilde{t}_{R}}^{2}+m_{t}^{2}-\frac{2}{3}\left(M_{W}^{2}-M_{Z}^{2}\right) c_{2 \beta}
\end{array}\right)
$$

where $h_{t}$ and $A_{t}$ are the top quark Yukawa coupling and trilinear soft parameter respectively, and $M_{W}^{2} c_{2 \beta}=g_{w}^{2}\left(v_{1}^{2}+u^{2}-v_{2}^{2}\right), M_{Z}^{2} c_{2 \beta}=g_{z}^{2}\left(v_{1}^{2}+u^{2}-v_{2}^{2}\right)$. Following the procedure described in Section II, we find the contributions from the stop mass eigenstates $\tilde{t}_{1,2}$ as follows:

$$
\begin{aligned}
& \Sigma_{L_{i}}^{(1)}\left(\tilde{t}_{j}\right)=\frac{6}{64 \pi^{2}}\left\{\frac{4 M_{D t}^{2}}{2 m_{\tilde{t}_{j}}^{2}-m_{\tilde{t}_{1}}^{2}-m_{\tilde{t}_{2}}^{2}} \frac{m_{t} \mu_{i}}{v^{2} s_{2 \beta} / 2}\right\} m_{\tilde{t}_{j}}^{2}\left(\frac{m_{\tilde{t}_{j}}^{2}}{Q^{2}}-1\right) \\
& \Sigma_{L_{i}}^{(2)}\left(\tilde{t}_{j}\right)=\frac{6}{64 \pi^{2}}\left\{\frac{1}{2} \frac{M_{Z}^{2}}{v^{2}}+\frac{M_{L t}^{2}-M_{R t}^{2}}{2 m_{\tilde{t}_{j}}^{2}-m_{\tilde{t}_{1}}^{2}-m_{\tilde{t}_{2}}^{2}} \frac{8 M_{W}^{2}-5 M_{Z}^{2}}{6 v^{2}}\right\} m_{\tilde{t}_{j}}^{2}\left(\frac{m_{\tilde{t}_{j}}^{2}}{Q^{2}}-1\right)
\end{aligned}
$$

where $M_{L t}^{2}=\mathcal{M}_{\tilde{t}, 11}^{2}, M_{D t}^{2}=\mathcal{M}_{\tilde{t}, 12}^{2}$ and $M_{R t}^{2}=\mathcal{M}_{\tilde{t}, 22}^{2}$ ignoring small R-parity violating contributions.

Sbottom contribution: The sbottom mass matrix is

$$
\mathcal{M}_{\tilde{b}}^{2}=\left(\begin{array}{cc}
m_{Q_{3}}^{2}+m_{b}^{2}-\frac{1}{6}\left(2 M_{W}^{2}+M_{Z}^{2}\right) c_{2 \beta} & -A_{b} v_{2}-h_{b} \mu v_{1}+A_{i}^{\prime} u_{i} \\
-A_{b} v_{2}-h_{b} \mu v_{1}+A_{i}^{\prime} u_{i} & m_{\tilde{b}_{R}}^{2}+m_{b}^{2}+\frac{1}{3}\left(M_{W}^{2}-M_{Z}^{2}\right) c_{2 \beta}
\end{array}\right)
$$

The sbottom contributions are

$$
\begin{aligned}
& \Sigma_{L_{i}}^{(1)}\left(\tilde{b}_{j}\right)=\frac{6}{64 \pi^{2}}\left\{2 \lambda_{i}^{\prime} \frac{m_{b}}{v_{1}}+\frac{4 M_{D b}^{2}}{2 m_{\tilde{b}_{j}}^{2}-m_{\tilde{b}_{1}}^{2}-m_{\tilde{b}_{2}}^{2}} \frac{A_{i}^{\prime}}{v_{1}}\right\} m_{\tilde{b}_{j}}^{2}\left(\frac{m_{\tilde{b}_{j}}^{2}}{Q^{2}}-1\right) \\
& \Sigma_{L_{i}}^{(2)}\left(\tilde{b}_{j}\right)=\frac{6}{64 \pi^{2}}\left\{-\frac{1}{2} \frac{M_{Z}^{2}}{v^{2}}+\frac{M_{L b}^{2}-M_{R b}^{2}}{2 m_{\tilde{b}_{j}}^{2}-m_{\tilde{b}_{1}}^{2}-m_{\tilde{b}_{2}}^{2}} \frac{-4 M_{W}^{2}+M_{Z}^{2}}{6 v^{2}}\right\} m_{\tilde{t}_{j}}^{2}\left(\frac{m_{\tilde{t}_{j}}^{2}}{Q^{2}}-1\right)
\end{aligned}
$$

where $M_{L b}^{2}, M_{R b}^{2}$, and $M_{D b}^{2}$ are defined as in the stop case. The contributions from the first two squark generations can be obtained by obvious substitutions. 
Charged slepton/Higgs contribution: Let us denote the charged slepton and Higgs contributions to $\Sigma$ 's as

$$
\Sigma_{L_{j}}^{(1,2)}(\phi)=\frac{4}{64 \pi^{2}} S_{j}^{(1,2)}(\phi) m_{\phi}^{2}\left(\ln \frac{m_{\phi}^{2}}{Q^{2}}-1\right),
$$

where $\phi$ stands for the mass eigenstates $\tilde{e}_{i 1}, \tilde{e}_{i 2}$ and $H^{-}$. Here $S_{j}^{(1)}(\phi) \equiv\left(\partial m_{\phi}^{2} / \partial u_{j}\right) / 2 v_{1}$ and $S_{j}^{(2)}(\phi) \equiv\left(\partial m_{\phi}^{2} / \partial u_{j}\right) / 2 u_{j}$ can be calculated from the diagonalization of the mass matrix ( 5.6). In the matrix (6), the R-parity conserving mass components are

$$
\begin{aligned}
& M_{L i}^{2}=m_{L_{i}}^{2}+m_{i}^{e 2}-\frac{1}{2}\left(2 M_{W}^{2}-M_{Z}^{2}\right) c_{2 \beta}, \\
& M_{D i}^{2}=-A_{i}^{e} v_{1}-h_{i}^{e} v_{2} \mu \\
& M_{R i}^{2}=m_{\tilde{e}_{i R}}^{2}+m_{i}^{e 2}+\left(M_{W}^{2}-M_{Z}^{2}\right) c_{2 \beta}
\end{aligned}
$$

apart from $u^{2}$ dependence in the gauge boson masses. The slepton mass diagonalization matrix elements $c_{e i}$ and $s_{e i}$ define the rotation angle as $\tan \theta_{e i}=s_{e i} / c_{e i}$ and useful quantities which are free from the ambiguity in defining the angle $\theta_{e i}$ are

$$
\sin 2 \theta_{e i}=\frac{-2 M_{D i}^{2}}{m_{\tilde{e}_{i 1}}^{2}-m_{\tilde{e}_{i 2}}^{2}}, \quad \cos 2 \theta_{e i}=\frac{M_{L i}^{2}-M_{R i}^{2}}{m_{\tilde{e}_{i 1}}^{2}-m_{\tilde{e}_{i 2}}^{2}}
$$

A straightforward calculation leads to

$$
\begin{aligned}
S_{j}^{(1)}\left(\tilde{e}_{i 1}, \tilde{e}_{i 2}\right)=\frac{\delta_{i j}}{2 v_{1}^{2} F_{i}}\left\{2 m_{i}^{e} v_{1} \lambda_{i} F_{i}-\left(m_{L_{i} H_{1}}^{2}+B_{i} t_{\beta}\right) c_{\beta}^{2} F_{i}\right. \\
\quad+\left(m_{L_{i} H_{1}}^{2} t_{\beta}-B_{i}\right)\left(\left(M_{R i}^{2}-m_{H^{-}}^{2}\right) M_{W^{2}}^{2} c_{\beta}^{2} s_{2 \beta}+M_{D i}^{4} \frac{s_{2 \beta}}{2}-m_{i}^{e} \mu M_{D i}^{2}\right) \\
\quad-\left(m_{i}^{e} \mu_{i}\right)\left(m_{i}^{e} \mu\left(M_{L i}^{2}-m_{H^{-}}^{2}\right) / c_{\beta}^{2}-M_{D i}^{2}\left(M_{L i}^{2}-m_{H^{-}}^{2}+2 M_{W}^{2} c_{\beta}^{2}\right) t_{\beta}\right) \\
\quad \pm \frac{M_{L i}^{2}-M_{R i}^{2}}{m_{\tilde{e}_{i 1}}^{2}-m_{\tilde{e}_{i 2}}^{2}}\left[-\left(m_{L_{i} H_{1}}^{2}+B_{i} t_{\beta}\right) c_{\beta}^{2} F_{i}+\left(m_{i}^{e} \mu_{i}\right)\left(m_{i}^{e} \mu\right)\left(M_{L i}^{2}-m_{H^{-}}^{2}\right) / c_{\beta}^{2}\right. \\
\left.\left.\quad+\left(m_{L_{i} H_{1}}^{2} t_{\beta}-B_{i}\right)\left(M_{R i}^{2}-m_{H^{-}}^{2}\right) M_{W}^{2} c_{\beta}^{2} s_{2 \beta}+M_{D i}^{4} \frac{s_{2 \beta}}{2}\right)\right] \\
\quad \pm \frac{2 M_{D i}^{2}}{m_{\tilde{e}_{i 1}}^{2}-m_{\tilde{e}_{i 2}}^{2}}\left[A_{i} v_{1} F_{i}+m_{i}^{e} \mu_{i} t_{\beta} F_{i}\right. \\
\quad-\frac{1}{2}\left(m_{i}^{e} \mu_{i}\right)\left(\left(M_{L i}^{2}+M_{R i}^{2}-2 m_{H^{-}}^{2}\right)\left(M_{L i}^{2}-m_{H^{-}}^{2}+2 M_{W}^{2} c_{\beta}^{2}\right) t_{\beta}-2 m_{i}^{e} \mu M_{D i}^{2} / c_{\beta}^{2}\right) \\
\left.\left.\quad+\frac{1}{2}\left(m_{L_{i} H_{1}}^{2} t_{\beta}-B_{i}\right)\left(m_{i}^{e} \mu\left(M_{L i}^{2}+M_{R i}^{2}-2 m_{H^{-}}^{2}\right)-M_{D i}^{2}\left(M_{L i}^{2}-m_{H^{-}}^{2}+2 M_{W}^{2} c_{\beta}^{2}\right) s_{2 \beta}\right)\right]\right\} \\
S_{i}^{(1)}\left(H^{-}\right)=-\frac{m_{L_{i} H_{1}}^{2} t_{\beta}-B_{i}}{v_{1}^{2}}\left(\left(M_{R i}^{2}-m_{H^{-}}^{2}\right) M_{W}^{2} c_{\beta}^{2} s_{2 \beta}+M_{D i}^{4} \frac{s_{2 \beta}}{2}+M_{D i}^{2} m_{i}^{e} \mu\right) \\
\quad+\frac{m_{i}^{e} \mu_{i}}{v_{1}^{2}}\left(m_{i}^{e} \mu\left(M_{L i}^{2}-m_{H^{-}}^{2}\right) / c_{\beta}^{2}-M_{D i}^{2}\left(M_{L i}^{2}-m_{H^{-}}^{2}+2 M_{W}^{2} c_{\beta}^{2}\right) t_{\beta}\right)
\end{aligned}
$$

where $F_{i} \equiv\left(m_{\tilde{e}_{i 1}}^{2}-m_{H^{-}}^{2}\right)\left(m_{\tilde{e}_{i 2}}^{2}-m_{H^{-}}^{2}\right)$.

Note that the contributions for the two light sleptons are reduced to the following simple form taking $m_{1,2}^{e}=0$ : 


$$
\begin{aligned}
& S_{i}^{(1)}\left(\tilde{e}_{i L}\right)=-\frac{m_{L_{i} H_{1}}^{2}+B_{i} t_{\beta}}{v^{2}}+\frac{m_{L_{i} H_{1}}^{2} t_{\beta}-B_{i}}{v^{2}} \frac{M_{W}^{2} s_{2 \beta}}{\left(M_{L i}^{2}-m_{H^{-}}^{2}\right)} \\
& S_{i}^{(1)}\left(\tilde{e}_{i R}\right)=0
\end{aligned}
$$

Similarly, one finds

$$
\begin{aligned}
& S_{j}^{(2)}\left(\tilde{e}_{i 1}, \tilde{e}_{i 2}\right)=-\frac{1}{4} \frac{M_{Z}^{2}}{v^{2}} \pm \frac{M_{L i}^{2}-M_{R i}^{2}}{m_{\tilde{e}_{i 1}}^{2}-m_{\tilde{e}_{i 2}}^{2}}\left(\frac{3}{4} \frac{M_{Z}^{2}}{v^{2}}-\frac{M_{W}^{2}}{v^{2}}\right) \\
& +\frac{1}{2} \frac{\delta_{i j}}{v_{1}^{2} F_{i}}\left\{\left(m_{H^{-}}^{2} s_{\beta}^{2}-M_{L i}^{2}-M_{W}^{2} c_{\beta}^{2}\right) F_{i}+m_{i}^{e 2} \mu^{2}\left(M_{L i}^{2}-m_{H^{-}}^{2}\right) / c_{\beta}^{2}\right. \\
& +\left(M_{R i}^{2}-m_{H^{-}}^{2}\right)\left(M_{L i}^{2}-m_{H^{-}}^{2}+2 M_{W}^{2} c_{\beta}^{2}\right)^{2} s_{\beta}^{2}-2 m_{i}^{e} \mu t_{\beta} M_{D i}^{2}\left(M_{L i}^{2}-m_{H^{-}}^{2}+2 M_{W}^{2} c_{\beta}^{2}\right) \\
& \pm \frac{M_{L i}^{2}-M_{R i}^{2}}{m_{\tilde{e}_{i 1}}^{2}-m_{\tilde{e}_{i 2}}^{2}}\left[\left(m_{H^{-}}^{2} s_{\beta}^{2}-M_{L i}^{2}-M_{W}^{2} c_{\beta}^{2}\right) F_{i}-m_{i}^{e 2} \mu^{2}\left(M_{L i}^{2}-m_{H^{-}}^{2}\right) / c_{\beta}^{2}\right. \\
& \left.\quad+\left(M_{R i}^{2}-m_{H^{-}}^{2}\right)\left(M_{L i}^{2}-m_{H^{-}}^{2}+2 M_{W}^{2} c_{\beta}^{2}\right)^{2} s_{\beta}^{2}\right] \\
& \pm \frac{2 M_{D i}^{2}}{m_{\tilde{e}_{i 1}}^{2}-m_{\tilde{e}_{i 2}}^{2}}\left[\left(-2 A_{i} v_{1}-M_{D i}^{2}\right) F_{i}-M_{D i}^{2}\left(m_{i}^{e 2} \mu^{2} / c_{\beta}^{2}+\left(M_{L i}^{2}-m_{H^{-}}^{2}+2 M_{W^{2}}^{2} c_{\beta}^{2}\right)^{2} s_{\beta}^{2}\right)\right. \\
& \left.\left.\quad+m_{i}^{e} \mu t_{\beta}\left(M_{L i}^{2}+M_{R i}^{2}-2 m_{H^{-}}^{2}\right)\left(M_{L i}^{2}-m_{H^{-}}^{2}+2 M_{W^{2}}^{2} c_{\beta}^{2}\right)\right]\right\} \\
& S_{i}^{(2)}\left(H^{-}\right)=\frac{1}{v^{2}}\left\{\left(M_{W}^{2}\left(1+2 s_{\beta}^{2}\right)-\frac{1}{2} M_{Z}^{2} c_{2 \beta}+\left(M_{L i}^{2}-m_{H^{-}}^{2}\right) t_{\beta}^{2}\right) F_{i}\right. \\
& \quad\left(M_{R i}^{2}-m_{H^{-}}^{2}\right)\left(M_{L i}^{2}-m_{H^{-}}^{2}+2 M_{W_{\beta}}^{2} c_{\beta}^{2}\right)^{2} s_{\beta}^{2}+m_{i}^{e 2} \mu^{2}\left(M_{L i}^{2}-m_{H^{-}}^{2}\right) / c_{\beta}^{2} \\
& \left.\quad+2 m_{i}^{e} \mu t_{\beta} M_{D i}^{2}\left(M_{L i}^{2}-m_{H^{-}}^{2}+2 M_{W}^{2} c_{\beta}^{2}\right)\right\}
\end{aligned}
$$

$C P$-even sneutrino/Higgs contribution: As in the above case, we write

$$
\Sigma_{L_{i}}^{(1,2)}(\phi)=\frac{2}{64 \pi^{2}} S_{i}^{(1,2)}(\phi) m_{\phi}^{2}\left(\ln \frac{m_{\phi}^{2}}{Q^{2}}-1\right),
$$

where $\phi$ runs for $\operatorname{Re}(\tilde{\nu}), h^{0}$ and $H^{0}$ and $S_{i}^{(1),(2)}(\phi)$ are calculated from the matrix (14) as follows:

$$
\begin{aligned}
S_{j}^{(1)}\left(\tilde{\nu}_{i}\right)= & -\frac{\delta_{i j}}{F_{i}} \frac{M_{Z}^{2}}{v^{2}}\left(m_{L_{i} H_{1}}^{2}\left(m_{A^{0}}^{2} c_{2 \beta}-m_{\tilde{\nu}_{i}}^{2}\right)+B_{i} t_{\beta}\left(m_{A^{0}}^{2} c_{2 \beta}+m_{\tilde{\nu}_{i}}^{2}\right)\right) \\
S_{i}^{(1)}\left(h^{0}, H^{0}\right)= & \frac{1}{2} \frac{M_{Z}^{2}}{v^{2}} \frac{1}{F_{i}}\left\{\left(m_{L_{i} H_{1}}^{2}\left(m_{A^{0}}^{2} c_{2 \beta}-m_{\tilde{\nu}_{i}}^{2}\right)+B_{i} t_{\beta}\left(m_{A^{0}}^{2} c_{2 \beta}+m_{\tilde{\nu}_{i}}^{2}\right)\right)\right. \\
& \mp c_{2 \alpha}\left(m_{L_{i} H_{1}}^{2}\left(m_{A^{0}}^{2} c_{\beta}^{2}+M_{Z}^{2} s_{\beta}^{2}-m_{\tilde{\nu}_{i}}^{2}\right)+B_{i} t_{\beta}\left(m_{A^{0}}^{2} s_{\beta}^{2}+M_{Z}^{2} c_{\beta}^{2}-m_{\tilde{\nu}_{i}}^{2}\right)\right) \\
& \left.\mp \frac{s_{2 \alpha}}{2}\left(\left(m_{L_{i} H_{1}}^{2} t_{\beta}+B_{i}\right)\left(m_{A^{0}}^{2}+M_{Z}^{2}\right) c_{2 \beta}+2\left(m_{L_{i} H_{1}}^{2} t_{\beta}-B_{i}\right) m_{\tilde{\nu}_{i}}^{2}\right)\right\} \\
S_{j}^{(2)}\left(\tilde{\nu}_{i}\right)= & \frac{1}{2} \frac{M_{Z}^{2}}{v^{2}}\left[1+\delta_{i j}\left(1+\frac{M_{Z}^{2}\left(m_{\tilde{\nu}_{i}}^{2}-m_{A^{0}}^{2} c_{2 \beta}^{2}\right)}{F_{i}}\right)\right] \\
S_{i}^{(2)}\left(h^{0}, H^{0}\right)= & \frac{1}{2} \frac{M_{Z}^{2}}{v^{2}}\left\{\frac{M_{Z}^{2}\left(m_{A^{0}}^{2} c_{2 \beta}^{2}-m_{\tilde{\nu}_{i}}^{2}\right)}{F_{i}} \mp c_{2 \alpha}\left[1+\frac{M_{Z}^{2}\left(m_{A^{0}}^{2}-m_{\tilde{\nu}_{i}}^{2}\right) c_{2 \beta}}{F_{i}}\right]\right. \\
& \left.\mp s_{2 \alpha} s_{2 \beta} \frac{M_{Z}^{2} m_{\tilde{\nu}_{i}}^{2}}{F_{i}}\right\}
\end{aligned}
$$


where $F_{i} \equiv m_{\tilde{\nu}_{i}}^{4}-2 m_{\tilde{\nu}_{i}}^{2}\left(m_{h^{0}}^{2}+m_{H^{0}}^{2}\right)+m_{h^{0}}^{2} m_{H^{0}}^{2}$, and $m_{\tilde{\nu}_{i}}^{2}=m_{L_{i}}^{2}+\frac{1}{2} M_{Z}^{2} c_{2 \beta}$.

Recall that

$$
c_{2 \alpha}=-c_{2 \beta} \frac{m_{A^{0}}^{2}-M_{Z}^{2}}{m_{H^{0}}^{2}-m_{h^{0}}^{2}}, \quad s_{2 \alpha}=-s_{2 \beta} \frac{m_{A^{0}}^{2}+M_{Z}^{2}}{m_{H^{0}}^{2}-m_{h^{0}}^{2}}
$$

CP-odd sneutrino/Higgs contribution: Again, one can calculate from the matrix (17),

$$
\Sigma_{L_{j}}^{(1,2)}(\phi)=\frac{2}{64 \pi^{2}} S_{i}^{(1,2)}(\phi) m_{\phi}^{2}\left(\ln \frac{m_{\phi}^{2}}{Q^{2}}-1\right),
$$

where $\phi$ runs for $\operatorname{Im}(\tilde{\nu}), A^{0}$. Here, $S_{i}^{(1),(2)}(\phi)$ are given by

$$
\begin{aligned}
S_{j}^{(1)}\left(\tilde{\nu}_{i}^{I}\right) & =-\delta_{i j} \frac{m_{L_{i} H_{1}}^{2}+B_{i} t_{\beta}}{v^{2}}, & S_{i}^{(1)}\left(A^{0}\right) & =0 \\
S_{j}^{(2)}\left(\tilde{\nu}_{i}^{I}\right) & =\frac{1}{2} \frac{M_{Z}^{2}}{v^{2}}-\delta_{i j} \frac{m_{\tilde{\nu}_{i}}^{2}}{v^{2}}, & S_{i}^{(2)}\left(A^{0}\right) & =-\frac{1}{2} \frac{M_{Z}^{2}}{v^{2}} c_{2 \beta}
\end{aligned}
$$

Gauge boson contribution:

$$
\begin{aligned}
& \Sigma_{L_{i}}^{(1)}(\phi)=0 \\
& \Sigma_{L_{i}}^{(2)}(\phi)=\frac{12}{64 \pi^{2}} \frac{M_{W}^{4}}{v^{2}}\left(\ln \frac{M_{W}^{2}}{Q^{2}}-1\right)+\frac{6}{64 \pi^{2}} \frac{M_{Z}^{4}}{v^{2}}\left(\ln \frac{M_{Z}^{2}}{Q^{2}}-1\right)
\end{aligned}
$$

Bottom quark contribution:

$$
\begin{aligned}
& \Sigma_{L_{i}}^{(1)}(\phi)=-\frac{24}{64 \pi^{2}} \frac{m_{b}}{v_{1}} \lambda_{i} \frac{m_{b}^{2}}{v^{2}}\left(\ln \frac{m_{b}^{2}}{Q^{2}}-1\right) \\
& \Sigma_{L_{i}}^{(2)}(\phi)=0
\end{aligned}
$$

Neutrino/neutralino contribution: To diagonalize the $7 \mathrm{x} 7$ neutrino/neutralino mass matrix, we use the seesaw formula to obtain the rotation matrix:

$$
\mathcal{U}^{N}=\left(\begin{array}{cc}
1 & \Theta_{N} \\
-\Theta_{N}^{\dagger} & 1
\end{array}\right)\left(\begin{array}{cc}
U^{\nu} & 0 \\
0 & N
\end{array}\right)
$$

where the seesaw diagonalization matrix $\Theta_{N}$ separating out the neutrino and neutralino parts is given by $\Theta_{N}=M_{D} M_{N}^{-1}$, and $U^{\nu}$ is the neutrino mixing matrix which is determined from the full neutrino mass matrices including the tree and one-loop contributions. The matrix $N$ is the usual neutralino diagonalization matrix whose analytic expression can be found, e.g., in the paper by Barger et al. in Ref. [14]. We present a simpler form as follows:

$$
\begin{aligned}
& \frac{N_{2 i}}{N_{1 i}}=-\frac{1}{t_{W}} \frac{m_{\chi_{i}^{0}}-M_{1}}{m_{\chi_{i}^{0}}-M_{2}} \\
& \frac{N_{3 i}}{N_{1 i}}=-\frac{1}{s_{W}} \frac{\left(m_{\chi_{i}^{0}}-M_{1}\right)\left(m_{\chi_{i}^{0}} c_{\beta}-\mu s_{\beta}\right)}{M_{Z}\left(m_{\chi_{i}^{0}}-\mu s_{2 \beta}\right)} \\
& \frac{N_{3 i}}{N_{1 i}}=-\frac{1}{s_{W}} \frac{\left(m_{\chi_{i}^{0}}-M_{1}\right)\left(m_{\chi_{i}^{0}} s_{\beta}-\mu c_{\beta}\right)}{M_{Z}\left(m_{\chi_{i}^{0}}-\mu s_{2 \beta}\right)}
\end{aligned}
$$


and the $N_{i j}$ satisfies the unitarity relation, $N_{1 i}^{2}+N_{2 i}^{2}+N_{3 i}^{2}+N_{4 i}^{2}=1$. Recall that one has to keep R-parity violating contributions to calculate $\Sigma$ 's which we do not show explicitly in, e.g., (A19).

The sneutrino VEVs contributions are

$$
\Sigma_{L_{i}}^{(1,2)}(\psi)=-\frac{8}{64 \pi^{2}} S_{i}^{(1,2)}(\psi) m_{\psi}^{2}\left(\ln \frac{m_{\psi}^{2}}{Q^{2}}-1\right)
$$

where $\psi$ runs for four neutralinos $\chi_{i}^{0}$, and $S^{(1,2)}(\psi)$ are given by

$$
\begin{aligned}
S_{i}^{(1)}(\psi) & =\mu_{i} \frac{M_{Z}^{2}}{v^{2}} m_{\psi}\left[-m_{\psi}^{2} t_{\beta}+m_{\psi}\left(M_{\tilde{\gamma}} t_{\beta}+\mu\right)-\mu M_{\tilde{\gamma}}\right] / D(\psi) \\
S_{i}^{(2)}(\psi) & =\frac{M_{Z}^{2}}{v^{2}} m_{\psi}\left[-m_{\psi}^{3}+m_{\psi}^{2} M_{\tilde{\gamma}}+m_{\psi} \mu^{2}-\mu^{2} M_{\tilde{\gamma}}\right] / D(\psi),
\end{aligned}
$$

where

$$
\begin{aligned}
D(\psi)= & 5 m_{\psi}^{4}-4 m_{\psi}^{3}\left(M_{1}+M_{2}\right)+3 m_{\psi}^{2}\left(M_{1} M_{2}-\mu^{2}-M_{Z}^{2}\right) \\
& +2 m_{\psi}\left[\mu^{2}\left(M_{1}+M_{2}\right)+M_{Z}^{2}\left(2 \mu s_{2 \beta}+M_{\tilde{\gamma}}\right)\right]+\operatorname{det}\left(M_{N}\right) .
\end{aligned}
$$

Note that $S_{i}^{(1)}=0$ in the basis with $\mu_{i}=0$.

Charged lepton/chargino contribution: Diagonalization matrices $\mathcal{U}^{R}, \mathcal{U}^{L}$ bring respectively the weak eigenstates $\left(e_{i}^{c}, \tilde{W}^{+}, \tilde{H}_{2}{ }^{+}\right)$and $\left(e_{i}, \tilde{W}^{-}, \tilde{H}_{1}{ }^{-}\right)$to the mass eigenstates $\left(e_{i}^{ \pm}, \chi_{1,2}^{ \pm}\right)$ and are given by

$$
\begin{aligned}
\mathcal{U}^{R} & =\left(\begin{array}{ccc}
1 & r_{1}^{i} c_{r}+r_{2}^{i} s_{r} & -r_{1}^{i} s_{r}+r_{2}^{i} c_{r} \\
-r_{1}^{i} & c_{r} & -s_{r} \\
-r_{2}^{i} & s_{r} & c_{r}
\end{array}\right) \\
\mathcal{U}^{L} & =\left(\begin{array}{ccc}
1 & l_{1}^{i} c_{l}+l_{2}^{i} s_{l} & -l_{1}^{i} s_{l}+l_{2}^{i} c_{l} \\
-l_{1}^{i} & c_{l} & -s_{l} \\
-l_{2}^{i} & s_{l} & c_{l}
\end{array}\right)
\end{aligned}
$$

where $r_{1,1}$ and $l_{1,2}$ are given by

$$
\begin{aligned}
r_{1}^{i}=\left(\frac{u_{i}}{v_{1}}-\frac{\mu_{i}}{\mu}\right) \frac{\sqrt{2} m_{i}^{e} \mu M_{W}\left(\mu-M_{2} t_{\beta}\right) c_{\beta}}{\left(M_{2} \mu+M_{W}^{2} s_{2 \beta}\right)^{2}}, & r_{2}^{i}=\left(\frac{u_{i}}{v_{1}}-\frac{\mu_{i}}{\mu}\right) \frac{m_{i}^{e} \mu\left(M_{2}^{2}+2 M_{W}^{2} c_{\beta}^{2}\right)}{\left(M_{2} \mu+M_{W}^{2} s_{2 \beta}\right)^{2}} \\
l_{1}^{i}=\left(\frac{u_{i}}{v_{1}}-\frac{\mu_{i}}{\mu}\right) \frac{\sqrt{2} \mu M_{W} c_{\beta}}{M_{2} \mu+M_{W}^{2} s_{2 \beta}}, & l_{2}^{i}=\left(\frac{u_{i}}{v_{1}}-\frac{\mu_{i}}{\mu}\right) \frac{M_{W}^{2} s_{2 \beta}}{M_{2} \mu+M_{W}^{2} s_{2 \beta}}+\frac{\mu_{i}}{\mu}
\end{aligned}
$$

and the useful combination of the usual chargino mixing angles are given by

$$
\begin{array}{ll}
c_{l} c_{r}=\frac{M_{2} m_{\chi_{1}^{-}}+\mu m_{\chi_{2}^{-}}}{m_{\chi_{1}^{-}}^{2}-m_{\chi_{2}^{-}}^{2}}, & c_{l} s_{r}=\frac{\sqrt{2} M_{W}\left(s_{\beta} m_{\chi_{1}^{-}}+c_{\beta} m_{\chi_{2}^{-}}\right)}{m_{\chi_{1}^{-}}^{2}-m_{\chi_{2}^{-}}^{2}} \\
s_{l} s_{r}=\frac{\mu m_{\chi_{1}^{-}}+M_{2} m_{\chi_{2}^{-}}}{m_{\chi_{1}^{-}}^{2}-m_{\chi_{2}^{-}}^{2}}, & s_{l} c_{r}=\frac{\sqrt{2} M_{W}\left(c_{\beta} m_{\chi_{1}^{-}}+s_{\beta} m_{\chi_{2}^{-}}\right)}{m_{\chi_{1}^{-}}^{2}-m_{\chi_{2}^{-}}^{2}} .
\end{array}
$$


Neglecting the contributions from the two light charged leptons by taking $m_{e, \mu}=0$, we get

$$
\Sigma_{L_{i}}^{(1,2)}(\psi)=-\frac{8}{64 \pi^{2}} S_{i}^{(1,2)}(\psi) m_{\psi}^{2}\left(\ln \frac{m_{\psi}^{2}}{Q^{2}}-1\right)
$$

where $\psi$ runs for $\tau$ and the charginos $\chi_{1,2}^{-}$and $S^{(1,2)}(\psi)$ are given by

$$
\begin{aligned}
& S_{i}^{(1)}(\psi)= \mu_{i} \frac{m_{\psi}^{2}}{v^{2}}\left[2 M_{W}^{2}\left(\mu-M_{2} t_{\beta}\right)\left(m_{\psi}^{2}-m_{\tau}^{2}\right)+\delta_{i 3} m_{\tau}^{2} \mu\left(2 M_{W}^{2}+\left(m_{\psi}^{2}-M_{2}^{2}\right) / c_{\beta}^{2}\right)\right] / D(\psi) \\
&-\lambda_{i} m_{\tau} \frac{m_{\psi}^{2}}{v_{1}}\left[\left(m_{\psi}^{2}-m_{\chi_{1}^{+}}^{2}\right)\left(m_{\psi}^{2}-m_{\chi_{2}^{+}}^{2}\right)\right] / D(\psi) \\
& S_{i}^{(2)}(\psi)= 2 \frac{m_{\psi}^{2}}{v^{2}}\left[m_{\psi}^{4} M_{W}^{2}-m_{\psi}^{2} M_{W}^{2}\left(\mu^{2}+2 M_{W}^{2} s_{\beta}^{2}+m_{\tau}^{2}\right)+m_{\tau}^{2} M_{W}^{2}\left(\mu^{2}+2 M_{W}^{2} s_{\beta}^{2}\right)\right. \\
&\left.\quad+\delta_{i 3} m_{\tau}^{2}\left\{m_{\psi}^{4} / 2 c_{\beta}^{2}+m_{\psi}^{2}\left(M_{W}^{2}\left(3-t_{\beta}^{2}\right)-M_{2}^{2} / c_{\beta}^{2}\right)-2 M_{W}^{2}\left(M_{2} \mu t_{\beta}-3 M_{W}^{2} s_{\beta}^{2}-\mu^{2}\right)\right\}\right] / D(\psi)
\end{aligned}
$$

where

$D(\psi)=5 m_{\psi}^{6}-4 m_{\psi}^{4}\left(m_{\chi_{1}^{-}}^{2}+m_{\chi_{2}^{-}}^{2}+m_{\tau}^{2}\right)+3 m_{\psi}^{2}\left[m_{\tau}^{2}\left(m_{\chi_{1}^{-}}^{2}+m_{\chi_{2}^{-}}^{2}\right)+m_{\chi_{1}^{-}}^{2} m_{\chi_{2}^{-}}^{2}\right]-2 m_{\tau}^{2} m_{\chi_{1}^{-}}^{2} m_{\chi_{2}^{-}}^{2}$.

\section{APPENDIX B: ONE-LOOP CORRECTIONS TO NEUTRINO MASSES}

Here we summarize the calculation of one-loop self-energy diagrams in the weak basis. A key ingredient in this procedure is to express the propagators inside loops in terms of the mass basis propagators which can be done by the use of the diagonalization matrices (13, 15, 18, A18, A22). Schematically, one can write the transformation from the weak basis propagators to the mass basis ones as follows:

$$
\langle\alpha \beta\rangle=\sum_{\psi} \mathcal{P}_{\alpha \beta}^{\psi}\langle\psi \psi\rangle
$$

where $\alpha$ and $\beta$ represent weak basis fields and $\psi$ mass eigenstates. For neutrino/neutralino, charged lepton/chargino, sneutrino/Higgs (scalar or pseudoscalar), and charged slepton/Higgs propagators, one finds

$$
\begin{gathered}
\mathcal{P}_{\rho_{1}^{0} \rho_{2}^{0}}^{\psi^{0}}=\mathcal{U}_{\rho_{1}^{0} \psi^{0}}^{N} \mathcal{U}_{\rho_{2}^{0} \psi^{0}}^{N}, \quad \mathcal{P}_{\rho_{1}^{-} \rho_{2}^{+}}^{\psi^{ \pm}}=\mathcal{U}_{\rho_{1}^{-} \psi^{-}}^{L} \mathcal{U}_{\rho_{2}^{+} \psi^{+}}^{R} \\
\mathcal{P}_{\varphi_{1}^{0} \varphi_{2}^{0}}^{\phi^{0}}= \pm \frac{1}{2} \mathcal{U}_{\varphi_{1}^{0} \phi^{0}}^{S, P} \mathcal{U}_{\varphi_{2}^{0} \phi^{0}}^{S, P}, \quad \mathcal{P}_{\varphi_{1}^{0} \varphi_{2}^{\phi^{0}}}^{\phi^{0}}=\frac{1}{2} \mathcal{U}_{\varphi_{1}^{0} \phi^{0}}^{S, P} \mathcal{U}_{\varphi_{2}^{0 *} \phi^{0}}^{S, P} \\
\mathcal{P}_{\varphi_{1}^{-} \varphi_{2}^{+}}^{\phi^{-}}=\mathcal{U}_{\varphi_{1}^{-} \phi^{-}}^{C} \mathcal{U}_{\varphi_{2}^{+} \phi^{+}}^{C}
\end{gathered}
$$

where $\rho$ 's and $\psi$ 's represent the neutrino/neutralino or charged lepton/chargino fields in the weak and mass basis, respectively; $\varphi^{0}$ is a complex sneutrino or Higgs field and $\phi^{0}$ is a scalar or pseudoscalar mass eigenstates; $\varphi^{-}$and $\phi^{-}$represent the charged slepton/Higgs fields in the weak and mass basis, respectively.

We first present $\Pi_{n}$ which gives most significant contributions to the one-loop neutrino self-energy. 


$$
\begin{aligned}
& \Pi_{\nu_{i} \nu_{j}}=\left\{-\frac{g^{2}}{32 \pi^{2}} \sum_{\psi^{0}, \phi^{0}} \mathcal{P}_{\tilde{Z} \tilde{Z}}^{\psi^{0}} \mathcal{P}_{\tilde{\nu} \tilde{\nu}}^{\phi^{0}} m_{\psi^{0}} B_{0}\left(p^{2}, m_{\psi^{0}}^{2}, m_{\phi^{0}}^{2}\right)\right. \\
& -\frac{g h_{i}}{16 \pi^{2}} \sum_{\psi^{-}, \phi^{-}} \mathcal{P}_{\tilde{W}^{-} e_{i}^{c}}^{\psi^{-}} \mathcal{P}_{\tilde{e}_{j} H_{1}^{+}}^{\phi^{-}} m_{\psi^{-}} B_{0}\left(p^{2}, m_{\psi^{-}}^{2}, m_{\phi^{-}}^{2}\right) \\
& -\frac{g \lambda_{i}}{16 \pi^{2}} \delta_{j 3} \sum_{\psi^{-}, \phi^{-}} \mathcal{P}_{\tilde{W}^{-} \tau^{c}}^{\psi^{-}}\left(\mathcal{P}_{\tilde{\tau}_{L} \tilde{\tau}_{L}^{*}}^{\phi^{-}}-\mathcal{P}_{\tilde{e}_{i L} \tilde{e}_{i L}^{*}}^{\phi^{-}}\right) m_{\psi^{-}} B_{0}\left(p^{2}, m_{\psi^{-}}^{2}, m_{\phi^{-}}^{2}\right) \\
& -\frac{h_{i} h_{j}}{16 \pi^{2}} \sum_{\psi^{-}, \phi^{-}} \mathcal{P}_{\tilde{H}_{1}^{-} e_{i}^{c}}^{\psi^{-}} \mathcal{P}_{H_{1}^{-} \tilde{e}_{j R}^{*}}^{\phi^{-}} m_{\psi^{-}} B_{0}\left(p^{2}, m_{\psi^{-}}^{2}, m_{\phi^{-}}^{2}\right) \\
& -\frac{h_{\tau} \lambda_{j}}{16 \pi^{2}} \delta_{i 3} \sum_{\psi^{-}, \phi^{-}}\left(\mathcal{P}_{\tau \tau^{c}}^{\psi^{-}} \mathcal{P}_{H_{1}^{-} \tilde{\tau}_{R}^{*}}^{\phi^{-}}+\mathcal{P}_{\tilde{H}_{1}{ }^{-} \tau^{c}}^{\psi^{-}} \mathcal{P}_{\tilde{\tau}_{L} \tilde{\tau}_{R}^{*}}^{\phi^{-}}\right) m_{\psi^{-}} B_{0}\left(p^{2}, m_{\psi^{-}}^{2}, m_{\phi^{-}}^{2}\right) \\
& -\frac{\lambda_{i} \lambda_{j}}{16 \pi^{2}} \sum_{\psi^{-}, \phi^{-}} \mathcal{P}_{\tau \tau^{c}}^{\psi^{-}} \mathcal{P}_{\tilde{\tau}_{L} \tilde{\tau}_{R}^{*}}^{\phi^{-}} m_{\psi^{-}} B_{0}\left(p^{2}, m_{\psi^{-}}^{2}, m_{\phi^{-}}^{2}\right) \\
& \left.-\frac{\lambda_{i}^{\prime} \lambda_{j}^{\prime}}{16 \pi^{2}} \sum_{k} \mathcal{P}_{\tilde{b}_{L} \tilde{b}_{R}^{*}}^{\tilde{b}_{k}} m_{b} B_{0}\left(p^{2}, m_{b}^{2}, m_{\tilde{b}_{k}}^{2}\right)\right\}+(i \leftrightarrow j)
\end{aligned}
$$

The contributions to $\Pi_{D}$ are

$$
\begin{aligned}
& \Pi_{\nu_{i} \tilde{B}}=-\frac{g^{2}}{16 \pi^{2}} \frac{t_{W}}{2} \sum_{\psi^{0}, \phi^{0}}\left[-\mathcal{P}_{\tilde{W}_{3} \tilde{H}_{1}^{0}}^{\psi^{0}} \mathcal{P}_{\tilde{\nu}_{i} H_{1}^{0}}^{\phi^{0}}+\mathcal{P}_{\tilde{W}_{3} \tilde{H}_{2}^{0}}^{\psi^{0}} \mathcal{P}_{\tilde{\nu}_{i} H_{2}^{0}}^{\phi^{0}}\right] m_{\psi^{0}}^{2} B_{0}\left(p^{2}, m_{\psi^{0}}^{2}, m_{\phi^{0}}^{2}\right) \\
& -\frac{g^{2}}{16 \pi^{2}} \frac{t_{W}}{\sqrt{2}} \sum_{\psi^{-}, \phi^{-}}\left[2 \mathcal{P}_{\tilde{W}^{-} e_{i}^{c}}^{\psi^{-}} \mathcal{P}_{\tilde{e}_{i L} \tilde{e}_{i R}^{*}}^{\phi^{-}}+\mathcal{P}_{\tilde{W}^{-} \tilde{H}_{2}^{+}}^{\psi^{-}} \mathcal{P}_{\tilde{e}_{i L} H_{2}^{+}}^{\phi^{-}}\right] m_{\psi^{-}}^{2} B_{0}\left(p^{2}, m_{\psi^{-}}^{2}, m_{\phi^{-}}^{2}\right) \\
& -\frac{g h_{i}}{16 \pi^{2}} \frac{t_{W}}{\sqrt{2}} \sum_{\psi^{-}, \phi^{-}}\left[-\mathcal{P}_{e_{i} e_{i}^{e^{c}}}^{\psi^{-}} \mathcal{P}_{\tilde{e}_{i L} H_{1}^{+}}^{\phi^{-}}-\mathcal{P}_{\tilde{H}_{1}^{-} e_{i}^{c}}^{\psi^{-}} \mathcal{P}_{H_{1}^{-} H_{1}^{+}}^{\phi^{-}}\right. \\
& \left.+2 \mathcal{P}_{\tilde{H}_{1}{ }^{-}{ }^{c}{ }_{i}^{c}}^{\psi^{-}} \mathcal{P}_{\tilde{e}_{i R} \tilde{e}_{i R}^{*}}^{\phi^{-}}+\mathcal{P}_{\tilde{H}_{1}^{-} \tilde{H}_{2}^{+}}^{\psi^{-}} \mathcal{P}_{\tilde{e}_{i R} H_{2}^{+}}^{\phi^{-}}\right] m_{\psi^{-}}^{2} B_{0}\left(p^{2}, m_{\psi^{-}}^{2}, m_{\phi^{-}}^{2}\right) \\
& -\frac{g \lambda_{i}}{16 \pi^{2}} \frac{t_{W}}{\sqrt{2}} \sum_{\psi^{-}, \phi^{-}} \mathcal{P}_{\tau_{\tau^{c}}}^{\psi^{-}}\left[2 \mathcal{P}_{\tilde{\tau}_{i R} \tilde{\tau}_{i R}^{*}}^{\phi^{-}}-\mathcal{P}_{\tilde{\tau}_{i L} \tilde{\tau}_{i L}^{*}}^{\phi^{-}}\right] m_{\psi^{-}}^{2} B_{0}\left(p^{2}, m_{\psi^{-}}^{2}, m_{\phi^{-}}^{2}\right) \\
& -\frac{g \lambda_{i}^{\prime}}{16 \pi^{2}} \frac{t_{W}}{3 \sqrt{2}} \sum_{k}\left[2 \mathcal{P}_{\tilde{b}_{i R} \tilde{b}_{i R}^{*}}^{\tilde{b}_{k}}+\mathcal{P}_{\tilde{b}_{i L} \tilde{b}_{i L}^{*}}^{\tilde{b}_{k}}\right] m_{b}^{2} B_{0}\left(p^{2}, m_{b}^{2}, m_{\tilde{b}_{k}}^{2}\right) \\
& \Pi_{\nu_{i} \tilde{W}_{3}}=-\frac{g^{2}}{16 \pi^{2}} \frac{1}{2} \sum_{\psi^{0}, \phi^{0}}\left[\mathcal{P}_{\tilde{W}_{3} \tilde{H}_{1}^{0}}^{\psi^{0}} \mathcal{P}_{\tilde{\nu}_{i} H_{1}^{0}}^{\phi^{0}}-\mathcal{P}_{\tilde{W}_{3} \tilde{H}_{2}^{0}}^{\psi^{0}} \mathcal{P}_{\tilde{\nu}_{i} H_{2}^{0}}^{\phi^{0}}\right] m_{\psi^{0}}^{2} B_{0}\left(p^{2}, m_{\psi^{0}}^{2}, m_{\phi^{0}}^{2}\right) \\
& -\frac{g^{2}}{16 \pi^{2}} \frac{1}{\sqrt{2}} \sum_{\psi^{-}, \phi^{-}}\left[\mathcal{P}_{\tilde{W}^{-} \tilde{H}_{2}^{+}}^{\psi^{-}} \mathcal{P}_{\tilde{e}_{i L} H_{2}^{+}}^{\phi^{-}}\right] m_{\psi^{-}}^{2} B_{0}\left(p^{2}, m_{\psi^{-}}^{2}, m_{\phi^{-}}^{2}\right) \\
& -\frac{g h_{i}}{16 \pi^{2}} \frac{1}{\sqrt{2}} \sum_{\psi^{-}, \phi^{-}}\left[-\mathcal{P}_{e_{i} e_{i}^{c}}^{\psi^{-}} \mathcal{P}_{\tilde{e}_{i R} H_{1}^{+}}^{\phi^{-}}-\mathcal{P}_{\tilde{H}_{1}^{-} e_{i}^{c}}^{\psi^{-}} \mathcal{P}_{H_{1}^{-} H_{1}^{+}}^{\phi^{-}}+\mathcal{P}_{\tilde{H}_{1}^{-} \tilde{H}_{2}^{+}}^{\psi^{-}} \mathcal{P}_{\tilde{e}_{i R} H_{2}^{+}}^{\phi^{-}}\right] m_{\psi^{-}}^{2} B_{0}\left(p^{2}, m_{\psi^{-}}^{2}, m_{\phi^{-}}^{2}\right) \\
& -\frac{g \lambda_{i}}{16 \pi^{2}} \frac{1}{\sqrt{2}} \sum_{\psi^{-}, \phi^{-}}\left[-\mathcal{P}_{\tau \tau^{c}}^{\psi^{-}} \mathcal{P}_{\tilde{\tau}_{i L} \tilde{\tau}_{i L}^{*}}^{\phi^{-}}\right] m_{\psi^{-}}^{2} B_{0}\left(p^{2}, m_{\psi^{-}}^{2}, m_{\phi^{-}}^{2}\right)
\end{aligned}
$$




$$
\begin{aligned}
& -\frac{g \lambda_{i}^{\prime}}{16 \pi^{2}} \frac{1}{\sqrt{2}} \sum_{k}\left[-\mathcal{P}_{\tilde{b}_{i L} \tilde{b}_{i L}^{*}}^{\tilde{b}_{k}}\right] m_{b}^{2} B_{0}\left(p^{2}, m_{b}^{2}, m_{\tilde{b}_{k}}^{2}\right) \\
& \Pi_{\nu_{i} \tilde{H}_{1}^{0}}=-\frac{g^{2}}{16 \pi^{2}} \frac{1}{2} \sum_{\psi^{0}, \phi^{0}}\left[-t_{W} \mathcal{P}_{\tilde{W}_{3} \tilde{B}}^{\psi^{0}} \mathcal{P}_{\tilde{\nu}_{i} H_{1}^{0}}^{\phi^{0}}+\mathcal{P}_{\tilde{W}_{3} \tilde{W}_{3}}^{\psi^{0}} \mathcal{P}_{\tilde{\nu}_{i} H_{1}^{0}}^{\phi^{0}}\right] m_{\psi^{0}}^{2} B_{0}\left(p^{2}, m_{\psi^{0}}^{2}, m_{\phi^{0}}^{2}\right) \\
& -\frac{g h_{i}}{16 \pi^{2}} \sum_{\psi^{-}, \phi^{-}}\left[-\mathcal{P}_{\tilde{W}^{-} e_{i}^{c}}^{\psi^{-}} \mathcal{P}_{\tilde{e}_{i L} \tilde{e}_{i L}^{*}}^{\phi^{-}}+\mathcal{P}_{\tilde{W}^{-} e_{i}^{c}}^{\psi^{-}} \mathcal{P}_{H_{1}^{-} H_{1}^{+}}^{\phi^{-}}\right] m_{\psi^{-}}^{2} B_{0}\left(p^{2}, m_{\psi^{-}}^{2}, m_{\phi^{-}}^{2}\right)
\end{aligned}
$$

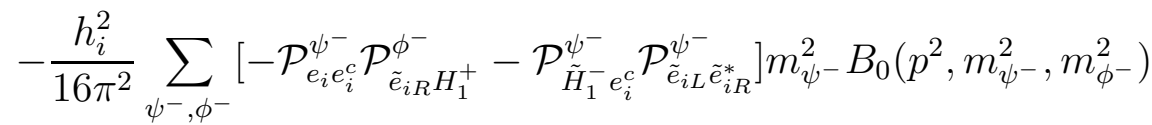

$$
\begin{aligned}
& -\frac{h_{\tau} \lambda_{i}}{16 \pi^{2}} \sum_{\phi^{-}}\left[-2 \mathcal{P}_{\tau_{i} \tau_{i}^{c}}^{\psi^{-}} \mathcal{P}_{\tilde{\tau}_{i L} \tilde{\tau}_{i R}^{*}}^{\phi^{-}}\right] m_{\psi^{-}}^{2} B_{0}\left(p^{2}, m_{\psi^{-}}^{2}, m_{\phi^{-}}^{2}\right) \\
& -\frac{h_{b} \lambda_{i}^{\prime}}{16 \pi^{2}} \sum_{k}\left[-2 \mathcal{P}_{\tilde{b}_{i L} \tilde{b}_{i R}^{*}}^{\tilde{b}_{k}}\right] m_{b}^{2} B_{0}\left(p^{2}, m_{b}^{2}, m_{\tilde{b}_{k}}^{2}\right) \\
& \Pi_{\nu_{i} \tilde{H}_{2}^{0}}=-\frac{g^{2}}{16 \pi^{2}} \frac{1}{2} \sum_{\psi^{0}, \phi^{0}}\left[t_{W} \mathcal{P}_{\tilde{W}_{3} \tilde{B}}^{\psi^{0}} \mathcal{P}_{\tilde{\nu}_{i} H_{2}^{0}}^{\phi^{0}}+\mathcal{P}_{\tilde{W}_{3} \tilde{W}_{3}}^{\psi^{0}} \mathcal{P}_{\tilde{\nu}_{i} H_{2}^{0}}^{\phi^{0}}\right] m_{\psi^{0}}^{2} B_{0}\left(p^{2}, m_{\psi^{0}}^{2}, m_{\phi^{0}}^{2}\right) \\
& -\frac{g^{2}}{16 \pi^{2}} \sum_{\psi^{-}, \phi^{-}}\left[\mathcal{P}_{\tilde{W}^{-} \tilde{W}^{+}}^{\psi^{-}} \mathcal{P}_{\tilde{e}_{i L} H_{2}^{+}}^{\phi^{-}}\right] m_{\psi^{-}}^{2} B_{0}\left(p^{2}, m_{\psi^{-}}^{2}, m_{\phi^{-}}^{2}\right) \\
& -\frac{g h_{i}}{16 \pi^{2}} \sum_{\psi^{-}, \phi^{-}}\left[\mathcal{P}_{\tilde{H}_{1}^{-} \tilde{W}^{+}}^{\psi^{-}} \mathcal{P}_{\tilde{e}_{i R} H_{2}^{+}}^{\phi^{-}}\right] m_{\psi^{-}}^{2} B_{0}\left(p^{2}, m_{\psi^{-}}^{2}, m_{\phi^{-}}^{2}\right)
\end{aligned}
$$

Note that $\mathcal{P}_{e_{i} e_{i}^{c}}^{\psi^{-}}$is equal to 1 for $\psi^{-}=e_{i}$ and vanishes for all others, and that $\mathcal{P}_{\tilde{b}_{L} \tilde{b}_{R}^{*}}^{\tilde{b}_{1,2}}=\mp c_{b} s_{b}$, $\mathcal{P}_{\tilde{b}_{L} \tilde{b}_{L}^{*}}^{\tilde{b}_{1,2}}=c_{b}^{2}, s_{b}^{2}$, and $\mathcal{P}_{\tilde{b}_{R} \tilde{b}_{R}^{*}}^{\tilde{b}_{1,2}^{*}}=s_{b}^{2}, c_{b}^{2}$ where $c_{b}$ and $s_{b}$ define the sbottom diagonalization angle; $\tan \theta_{b}=s_{b} / c_{b}$ as in Eq. (A7). The function $B_{0}$ is defined by

$$
B_{0}\left(p^{2}, m_{1}^{2}, m_{2}^{2}\right)=-\frac{m_{2}^{2}}{m_{1}^{2}-m_{2}^{2}} \ln \frac{m_{1}^{2}}{m_{2}^{2}}-\ln \frac{m_{1}^{2}}{Q^{2}}+1 \quad \text { with } \quad p^{2} \rightarrow 0 .
$$

\section{APPENDIX C: RGE IN THE BASIS WITH NO BILINEAR TERMS}

Renormalization group equations for the lepton number violating parameters in the basis where the bilinear terms $\mu_{i} L_{i} H_{1}$ in the superpotential are rotated away have been calculated in the second paper of Ref. [20]. Here we collect the results with a few minor corrections.

$$
\begin{aligned}
16 \pi^{2} \frac{d \lambda_{i}^{\prime}}{d t} & =\lambda_{i}^{\prime}\left(\delta_{i 3} h_{\tau}^{2}+h_{t}^{2}+3 h_{b}^{2}-\frac{7}{9} g^{\prime 2}-3 g_{2}^{2}-\frac{16}{3} g_{3}^{2}\right) \\
16 \pi^{2} \frac{d \lambda_{i}}{d t} & =\lambda_{i}\left(3 h_{\tau}^{2}-3 g^{\prime 2}-3 g_{2}^{2}\right) \\
16 \pi^{2} \frac{d A_{i}^{\prime}}{d t} & =A_{i}^{\prime}\left(\delta_{i 3} h_{\tau}^{2}+h_{t}^{2}+9 h_{b}^{2}-\frac{7}{9} g^{\prime 2}-3 g_{2}^{2}-\frac{16}{3} g_{3}^{2}\right)+A_{i}\left(2 h_{b} h_{\tau}\right) \\
& +2 \lambda_{i}^{\prime}\left(\delta_{i 3} h_{\tau} A_{\tau}+h_{t} A_{t}+3 h_{b} A_{b}+\frac{7}{9} g^{\prime 2} M_{1}+3 g_{2}^{2} M_{2}+\frac{16}{3} g_{3}^{2} M_{3}\right)
\end{aligned}
$$




$$
\begin{aligned}
16 \pi^{2} \frac{d A_{i}}{d t} & =A_{i}\left(5 h_{\tau}^{2}-3 g^{\prime 2}-3 g_{2}^{2}\right)+A_{i}^{\prime}\left(6 h_{b} h_{\tau}\right)+\lambda_{i}\left(6 h_{\tau} A_{\tau}+6 g^{\prime 2} M_{1}+6 g_{2}^{2} M_{2}\right) \\
16 \pi^{2} \frac{d m_{L_{i} H_{1}}^{2}}{d t} & =m_{L_{i} H_{1}}^{2}\left(\delta_{i 3} h_{\tau}^{2}+h_{\tau}^{2}+3 h_{b}^{2}\right)-6 A_{i}^{\prime} A_{b}-2 A_{i} A_{\tau} \\
& -6 \lambda_{i}^{\prime} h_{b}\left(m_{L_{i}}^{2}+m_{Q_{3}}^{2}+m_{D_{3}}^{2}\right)-2 \lambda_{i} h_{\tau}\left(m_{L_{i}}^{2}+m_{L_{3}}^{2}+m_{E_{3}}^{2}\right) \\
16 \pi^{2} \frac{d B_{i}}{d t} & =B_{i}\left(\delta_{i 3} h_{\tau}^{2}+3 h_{t}^{2}-g^{\prime 2}-3 g_{2}^{2}\right)-\mu\left(6 A_{i}^{\prime} h_{b}+2 A_{i} h_{\tau}\right)
\end{aligned}
$$

Note that these equations are valid when the R-parity violating couplings are small enough so that the next leading corrections are negligible. 


\section{REFERENCES}

[1] L. Hall and M. Suzuki, Nucl. Phys. B231 (1984) 419.

[2] A. S. Joshipura and M. Nowakowski, Phys. Rev. D51, 2421 (1995); M. Nowakowski and A. Pilaftsis, Nucl. Phys. B461, 19 (1996); F. M. Borzumati, Y. Grossman, E. Nardi and Y. Nir, Phys. Lett. B384 (1996) 123; B. de Carlos and P. L. White, Phys. Rev. D54 (1996) 3427; A. Yu. Smirnov and F. Vissani, Nucl. Phys. B460 (1996) 37; R. Hempfling, Nucl. Phys B478 (1996) 3; H. P. Nilles and N. Polonsky, Nucl. Phys. B484 (1997) 33; E. Nardi, Phys. Rev. D55 (1997) 5772.

[3] E. J. Chun, S. K. Kang, C. W. Kim and U. W. Lee, Nucl. Phys. B544, 89 (1999), hep-ph/9807327; A. S. Joshipura and S. K. Vempati, hep-ph/9808232; hep-ph/9903435; J. Ferrandis, hep-ph/9810371; M. Bisset, O. Kong, C. Macesanu and L. Orr, hepph/9811498; S. Rakshit, G. Bhattacharyya, Phys. Rev. D59, 091701 (1999); R. Adhikari, G. Omanovic, Phys. Rev. D59, 073003 (1999); D. E. Kaplan and A. Nelson, hep-ph/9901254; A. Abada and M. Losada, hep-ph/9908352; J. L. Chkareuli, et al., hep-ph/9908451; J. C. Romao, et al., hep-ph/9907499; O. Haug, et al., hep-ph/9909318.

[4] Super-Kamiokande Collaboration, Y. Fukuda, et.al., Phys. Rev. Lett. 81, 1562 (1998).

[5] B. T. Cleveland et al., Astrophys. J. 496, 505 (1998); Kamiokande Collaboration, K. S. Hirata et al., Phys. Rev. Lett. 77, 1683 (1996); GALLEX Collaboration, W. Hampel et al., Phys. Lett. B447, 127 (1999); SAGE Collaboration, J. N. Abdurashitov et al., astro-ph/9907113; Super-Kamiokande Collaboration, Y. Fukuda, et al., Phys. Rev. Lett. 82, 2430 (1999).

[6] LSND Collaboration, C. Athanassopoulos et al., Phys. Rev. Lett. 81, 1774 (1998).

[7] CHOOZ Collaboration, M. Apollonio et al., Phys. Lett. B420, 397 (1998); hepex/9907037.

[8] B. Mukhopadhyaya, S. Roy and F. Vissani, Phys. Lett. B443, 191 (1998).

[9] E. J. Chun and J. S. Lee, Phys. Rev. D60, 075006 (1999), hep-ph/9811201; S. Y. Choi, E. J. Chun, S. K. Kang and J. S. Lee, Phys. Rev. D60, 075002 (1999), hep-ph/9903465.

[10] S. Kiyoura, M. M. Nojiri, D. M. Pierce and Y. Yamada, Phys. Rev. D58, 075002 (1998).

[11] R. Hempfling, in Ref. [2].

[12] J. C. Romao, et al., in Ref. [3].

[13] V. Ben-Hamo and Y. Nir, Phys. Lett. B339, 77 (1994); T. Banks, Y. Grossman, E. Nardi and Y. Nir, Phys. Rev. D52, 5319 (1995); E. J. Chun and A. Lukas, Phys. Lett. B387, 99 (1996); K. Choi, et al., Phys. Lett. B394, 89 (1997); Phys. Rev. D60, 031301 (1999); G. Eyal and Y. Nir, JHEP 9906, 025 (1999), hep-ph/9904473; G. K. Leontaris and J. Rizos, hep-ph/9909206; Q. Shafi and Z. Tavartkiladze, hep-ph/9909238.

[14] R. Arnowitt and P. Nath, Phys. Rev. D46, 3981 (1992); V. Barger, M. Berger and P. Ohmann, Phys. Rev. D49, 4098 (1994).

[15] M. Nowakowski and A. Pilaftsis, in Ref. [2];

[16] D. Pierce and A. Papadopoulos, Nucl. Phys. B430, 278 (1994); Phys. Rev. D50, 565 (1995).

[17] M. Dine and A. Nelson, Phys. Rev. D48, 1277 (1993); M. Dine, A. Nelson and Y. Shirman, Phys. Rev. D51, 1362 (1995); M. Dine, A. Nelson, Y. Nir and Y. Shirman, Phys. Rev. D53, 1658 (1996).

[18] For a review, see G. F. Giudice and R. Rattazzi, hep-ph/9801271. 
[19] S. Dimopoulos, S. Thomas and J. D. Wells, Nucl. Phys. B488, 39 (1997).

[20] B. de Carlos and P. L. White, in Ref. [2]; E. J. Chun, el al., in Ref. [3].

[21] K. Choi, K. Hwang and E. J. Chun, Phys. Rev. D60, 031301 (1999); D. E. Kaplan and A. Nelson, in Ref. [3].

[22] For a recent analysis, see J. N. Bahcall, P. I. Krastev and A. Yu. Smirnov, Phys. Rev. D58, 096016 (1998).

[23] V. Barger et al., Phys. Lett. B437, 107 (1998); H. Georgi and S. L. Glashow, hepph/9808293; A. J. Baltz et al., Phys. Rev. Lett. 81, 5730 (1998); S. Davidson and S. F. King, Phys. Lett. B445, 191 (1998); C. Giunti, Phys. Rev. D59, 077301 (1999); Y. Nomura and T. Yanagida, Phys. Rev. D59, 017303 (1999); R. N. Mphapatra and S. Nussinov, hep-ph/9808301; M. Tanimoto, hep-ph/9807517; H. Fritzsch and Z. Xing, Phys. Lett. B440, 313 (1998); S. K. Kang and C. S. Kim, Phys. Rev. D59, 091302 (1999); C. S. Kim and J. D. Kim, hep-ph/9908435; C. Jarlskog et al., Phys. Lett. B449, 240 (1999); R. Barieri et al., hep-ph/9901241; Q.Shafi and A. Tavartkiladze, Phys. Lett. B451, 129 (1999); A. H. Guth et al., JHEP 9908, 018 (1999); J. Ellis and S. Lola, Phys. Lett. B458, 310 (1999); M. Jezabek and Y. Sumino, Phys. Lett. B457, 139 (1999); J. A. Casas et al., Nucl. Phys. B556, 3 (1999); Y. -L. Wu, hep-ph/9905222; D. Falcone, hep-ph/9905316; G. Altarelli et al., hep-ph/9905536; A. Ghosal, hep-ph/9905470; C. H. Albright and S. M. Barr, hep-ph/9906297. 


\section{TABLES}

TABLE I. R-parity violating parameters $m_{L_{i} H_{1}}^{2}, B_{i}$, and and one-loop corrections $\Sigma_{L_{i}}$ in the $\mathrm{GeV}$ unit. Each collum corresponds to the lepton generation, $i=1,2,3$. $\xi_{i}$ are one-loop improved sneutrino VEVs and $\xi_{i}^{0}$ are tree values in the unit of $v_{1}$. The last five rows show typical sizes of some components of tree and loop mass neutrino matrices and their eigenvalues in the eV unit.

\begin{tabular}{|c|c|c|c|c|c|c|}
\hline & \multicolumn{3}{|c|}{$\tan \beta=3$} & \multicolumn{3}{|c|}{$\tan \beta=30$} \\
\hline$m_{L_{i} H_{1}}^{2}$ & $4.00 \times 10^{-2}$ & $4.00 \times 10^{-2}$ & $3.93 \times 10^{-2}$ & 3.67 & 3.67 & 3.59 \\
\hline$B_{i} \tan \beta$ & $-3.95 \times 10^{-3}$ & $-3.95 \times 10^{-3}$ & $-3.88 \times 10^{-3}$ & -3.60 & -3.60 & -3.52 \\
\hline$\tilde{q}$ & $4.93 \times 10^{-3}$ & $4.93 \times 10^{-3}$ & $4.93 \times 10^{-3}$ & $5.02 \times 10^{-1}$ & $5.02 \times 10^{-1}$ & $5.00 \times 10^{-1}$ \\
\hline$\phi^{-}$ & $1.39 \times 10^{-3}$ & $1.39 \times 10^{-3}$ & $1.45 \times 10^{-3}$ & $-1.73 \times 10^{-2}$ & $-1.73 \times 10^{-2}$ & $-3.89 \times 10^{-2}$ \\
\hline$S^{0}$ & $-5.64 \times 10^{-4}$ & $-5.64 \times 10^{-4}$ & $-5.02 \times 10^{-4}$ & $-5.03 \times 10^{-2}$ & $-5.03 \times 10^{-2}$ & $-4.61 \times 10^{-2}$ \\
\hline$P^{0}$ & $7.21 \times 10^{-4}$ & $7.21 \times 10^{-4}$ & $7.55 \times 10^{-4}$ & $1.48 \times 10^{-3}$ & $1.48 \times 10^{-3}$ & $1.39 \times 10^{-3}$ \\
\hline$\Sigma_{L_{i}}^{(1)}$ & $6.47 \times 10^{-3}$ & $6.47 \times 10^{-3}$ & $6.63 \times 10^{-3}$ & $4.36 \times 10^{-1}$ & $4.36 \times 10^{-1}$ & $4.17 \times 10^{-1}$ \\
\hline$m_{\tilde{\nu}_{i}}^{2}$ & 57280 & 57280 & 62860 & 56440 & 56440 & 61340 \\
\hline$\tilde{q}$ & -4.8 & -4.84 & -4.84 & -6.22 & -6.22 & -6.22 \\
\hline$\phi^{-}$ & 2507 & 2507 & 2867 & 2382 & 2382 & 2178 \\
\hline$S^{0}$ & -356.3 & -356.3 & -360.2 & -329.8 & -329.8 & -331.9 \\
\hline$P^{0}$ & 739.6 & 739.6 & 935.6 & 759.3 & 759.3 & 928.9 \\
\hline$\psi^{0}$ & -712.6 & -712.6 & -712.6 & -486.3 & -486.3 & -486.3 \\
\hline$\psi^{-}$ & 782.8 & 782.8 & 787.3 & 745.0 & 745.0 & 1048 \\
\hline$Z / W$ & -258.8 & -258.8 & -258.8 & -258.8 & -258.8 & -258.8 \\
\hline$\Sigma_{L_{i}}^{(2)}$ & 2696 & 2696 & 3253 & 2805 & 2805 & 3072 \\
\hline$\frac{L_{i}}{\xi_{i}}$ & $-7.09 \times 10^{-7}$ & $-7.09 \times 10^{-7}$ & $-6.35 \times 10^{-7}$ & $-8.61 \times 10^{-6}$ & $-8.61 \times 10^{-6}$ & $-7.50 \times 10^{-6}$ \\
\hline$\xi_{i}^{0}$ & $-6.30 \times 10^{-7}$ & $-6.30 \times 10^{-7}$ & $-5.63 \times 10^{-7}$ & $-1.32 \times 10^{-6}$ & $-1.32 \times 10^{-6}$ & $-1.08 \times 10^{-6}$ \\
\hline tree mass & & $2 \times 10^{-3}$ & & & $3 \times 10^{-3}$ & \\
\hline$\Pi_{D}: \eta$ & & $10^{-5}$ & & & $10^{-4}$ & \\
\hline$\Pi_{n}: h \lambda$ & & $10^{-8}$ & & & $10^{-2}$ & \\
\hline$\Pi_{n}: \lambda \lambda$ & & $10^{-5}$ & & & $10^{-2}$ & \\
\hline igenvalues & 0 & $1 \times 10^{-5}$ & $7 \times 10^{-3}$ & 0 , & $7 \times 10^{-3}$ & $2 \times 10^{-2}$ \\
\hline
\end{tabular}

